Supporting Information

\title{
Sustainable Nanosheet Antioxidants for Sepsis Therapy via Scavenging Intracellular Reactive Oxygen and Nitrogen Species
}

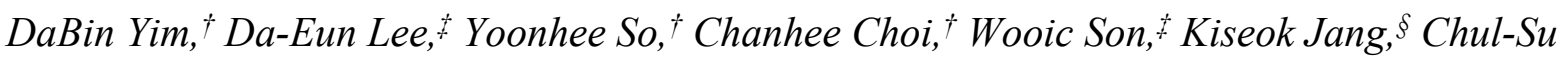
Yang, $, *, *$ and Jong-Ho Kim ${ }^{\dagger} *$

$\uparrow$ Department of Chemical Engineering, Hanyang University, Ansan 15588, Republic of Korea

\$ Department of Molecular and Life Science, Hanyang University, Ansan 15588, Republic of Korea

$\S$ Department of Pathology, Hanyang University College of Medicine, Seoul 04763, Republic of Korea

\# Department of Bionano Technology, Hanyang University, Seoul 04763, Republic of Korea

*Corresponding author: kjh75@hanyang.ac.kr, chulsuyang@hanyang.ac.kr 


\section{Materials}

All chemicals were used without further purification. For all experiments, sterilized distilled water was used. $\mathrm{PCL}_{460}-b-\mathrm{PEG}_{5000}$, in which the numbers denote the molecular weight of each block, was purchased from Advanced Polymer Materials Inc. (Canada). Tungsten disulfide $\left(\mathrm{WS}_{2}\right)$ powder, terephthalic acid, iron(II) sulfate heptahydrate, IR783, azino-bis(3ethylbenzthiazoline-6-sulphonic acid) (ABTS), and 6-hydroxy-2,5,7,8-tetramethychroman-2carboxylic acid (Trolox) were purchased from Sigma Aldrich. Molybdenum diselenide ( $\left.\mathrm{MoSe}_{2}\right)$ and tungsten diselenide $\left(\mathrm{WSe}_{2}\right)$ powders were purchased from Alfa Aesar. Nitric oxide assay kit was purchased from Abcam, and 5-(2,4-disulfophenyl)-3-(2-methoxy-4-nitrophenyl)-2-(4nitrophenyl)-2H-tetrazolium (WST-8) was purchased from Dojindo (Japan). p22phox (FL195), gp91phox (H-60), p47phox (H-195), UQCRC2 (G-10), and actin (I-19) were purchased from Santa Cruz Biotechnology. UQCRQ (PA5-106446), NDUFB8 (459210), and NDUFA9 (459100) were purchased from Invitrogen. Ethanol, hydrogen peroxide (30\%), and sodium nitroprusside were purchased from DAEJUNG chemicals \& metal Co., LTD (Korea), and phosphate-buffered saline (PBS) solution was purchased from Gibco. Wild-type C57BL/6 mice were purchased from Samtako Bio Korea (Gyeonggi-do, Korea).

\section{Instrument}

The morphology and structure of the TMD nanosheets were characterized by transmission electron microscopy (TEM, JEM-2100F, JEOL/CEOS, Japan), atomic force microscopy (AFM, XE-100, Park systems, Korea), and Fourier-transform infrared spectroscopy (Nicolet iS10, Thermo Fisher Scientific, USA). UV/Vis absorption spectra were measured by a UV/Vis spectrometer (Mega2100, Scinco, Korea) and a microplate reader (Power Wave XS, BioTek, USA). A spectrofluorometer (NanoLog, HORIBA, Japan) was used to measure the photoluminescence of a ROS indicator. All assays were conducted in a thermomixier 
(Eppendorf, Germany). Raman spectra were measured with a Raman microscope (UniRAM, Uni Nanotech., Korea) under excitation at $532 \mathrm{~nm}$. X-ray photoelectron spectroscopy (XPS, AXIS-His, KRATOS, UK) was used to confirm the oxidation states of $\mathrm{W}$, Mo, and $\mathrm{N}$ atoms. The concentration of TMD nanosheets was measured by inductive coupled plasma-atomic emission spectroscopy (ICP-AES, SPECTRO, SPECTRO ARCOS). The cell images were obtained by a high-voltage electron microscope (HVEM, JEM ARM 1300S, JEOL Ltd., Japan).

Calculation of the molecular weight of TMD nanosheets. $\mathrm{WS}_{2}$ nanosheet was used as a representative for the calculation of its molecular weight (MW). The average size of $\mathrm{WS}_{2}$ nanosheets was measured by TEM. The number of $\mathrm{W}$ atoms in a single $\mathrm{WS}_{2}$ nanosheet was calculated based on its lateral size of $37.5 \mathrm{~nm}$ and a lattice space of $0.27 \mathrm{~nm}$. The distance between $\mathrm{W}-\mathrm{W}$ was calculated to be $0.311 \mathrm{~nm}$ from the lattice space. This calculation found that a single $\mathrm{WS}_{2}$ nanosheet of $37.5 \mathrm{~nm}$ has $15,000 \mathrm{~W}$ atoms.

Calculation of the equilibrium constants for scavenging $\left(K_{s}\right)$ by TMD nanosheets. The equilibrium constants for scavenging of ROS and RNS by of TMD nanosheets were calculated using the Langmuir isotherm. A fractional coverage $\theta$ is shown below:

$$
\theta=\frac{K \cdot p}{1+K \cdot p}
$$

$K$ is an association constant where $K=\frac{k_{a}}{k_{d}}, k_{a}$ is an adsorption rate constant and $k_{d}$ is a desorption rate constant, and $p$ is pressure. For calculation of the equilibrium constants for ROS and RNS scavenging, the fractional coverage corresponds to $\mathrm{I} / \mathrm{I}_{\infty}$ where $\mathrm{I}$ is the ROS or RNS scavenging activity of TMD nanosheets at a certain concentration and $\mathrm{I}_{\infty}$ is their saturated ROS or RNS scavenging activity. In addition, the pressure is equivalent to the concentration of TMD nanosheets, $\mathrm{C}$, which gives the following equation: 


$$
\begin{aligned}
& \frac{I}{I_{\infty}}=\frac{K \cdot C}{1+K \cdot C} \\
& \frac{C}{I}=\frac{C}{I_{\infty}}+\frac{1}{K \cdot I_{\infty}}
\end{aligned}
$$

The above equation was applied to the plot of the ROS or RNS scavenging activity with the concentration of TMD nanosheets. By obtaining a slope and a y intercept, the equilibrium constants for ROS or RNS scavenging $\left(K_{\mathrm{s}}\right)$ by TMD nanosheets were calculated.

Sustainability of TMD nanosheets for hydroxyl radicals and nitric oxide. For hydroxyl radicals, $50 \mu \mathrm{L}$ of $\mathrm{H}_{2} \mathrm{O}_{2}(12 \mathrm{mM})$ and $50 \mu \mathrm{L}$ of $\mathrm{FeSO}_{4}(120 \mu \mathrm{M})$ were mixed for 30 min to produce hydroxyl radicals. The TMD nanosheets exfoliated with PCL- $b$-PEG (300 $\mu \mathrm{L}, 67 \mathrm{nM})$ were added to the hydroxyl radical solution, followed by shaking the mixture for $1 \mathrm{~h}$ at $25^{\circ} \mathrm{C}$. TA $(50 \mu \mathrm{L}, 30 \mathrm{mM}$, in $0.1 \mathrm{M} \mathrm{NaOH})$ was added to the TMD mixture, followed by shaking for further $30 \mathrm{~min}$. After the TMD nanosheets were settled down by centrifugation, the fluorescence of the supernatant was measured by a spectrofluorometer. Then, the obtained TMD nanosheets were washed twice with D.I water by centrifugation and they were reused for scavenging fresh hydroxyl radicals under the same condition.

For nitric oxide, it was produced by sodium nitroprusside (SNP). After scavenging, its amount was measured using a commercial assay kit (Abcam). $75 \mu \mathrm{L}$ of SNP $(8 \mathrm{mM})$ and $225 \mu \mathrm{L}$ of the TMD nanosheets $(67 \mathrm{nM})$ were mixed together in $0.1 \mathrm{M}$ PBS ( $\mathrm{pH} 7.4)$, followed by shaking for $30 \mathrm{~min}$. Then, $3.5 \mu \mathrm{L}$ of nitrate reductase and $3.5 \mu \mathrm{L}$ of an enzyme cofactor were added to the mixture. After reaction for $30 \mathrm{~min}, 35 \mu \mathrm{L}$ of Griess reagent $\mathrm{R} 1$ and $\mathrm{R} 2$ was added to the mixture solution, followed by shaking for further $30 \mathrm{~min}$. After the TMD nanosheets were settled down by centrifugation, the absorption of the supernatant was measured by a UV-Vis 
spectrometer. Then, the settled-down TMD nanosheets were washed twice with D.I water by centrifugation and they were reused for scavenging fresh nitric oxide under the same condition.

Mice and cell culture. Mouse primary bone marrow derived-macrophages (BMDMs) were isolated from $\mathrm{C} 57 \mathrm{BL} / 6$ mice and cultured in DMEM for three to five days in the presence of $25 \mathrm{ng} \mathrm{mL}^{-1}$ recombinant macrophage colony stimulating factor (R\&D Systems, 416-ML, Minneapolis, MN, USA), as described previously. ${ }^{1}$ The culture medium was Dulbecco's modified Eagle's medium (DMEM; Life Technologies) containing $4 \mathrm{mM}$ glutamine and 10\% fetal bovine serum (FBS; Life Technologies). Adherent primary monocytes were prepared from peripheral blood mononuclear cells donated by healthy subjects, as previously described. ${ }^{1}$ This study was approved by the Institutional Review Board of the Korean Red Cross, which oversees studies that use samples from human subjects.

MTT assay. Cell viability relative to non-treated group was measured by MTT (3-(4,5dimethylthiazol-2-yl)-2,5-diphenyltetrazolium bromide) assay. After incubating for indicated time points with TMDs in BMDMs, $5 \mathrm{mg} \mathrm{mL}^{-1}$ of MTT solution was added in the place of media, and cells were incubated for further $4 \mathrm{~h}$. Then, all the media was removed and the same volume of dimethyl sulfoxide (DMSO) solution was added for $15 \mathrm{~min}$ to dissolve the formazan. Using a UV/VIS spectrophotometer, each well of the plate was measured at $540 \mathrm{~nm}$ to assess relative cell viability.

Immunoblot analysis. The polypeptides were resolved by SDS-polyacrylamide gel electrophoresis (PAGE) and transferred to a PVDF membrane (Bio-Rad). Immuno detection was achieved with specific antibodies. Antibody binding was visualized by chemiluminescence (ECL; Millipore) and detected by a Vilber chemiluminescence analyzer (Fusion SL 3; Vilber Lourmat), as described previously. ${ }^{2}$ 
Enzyme-linked immunosorbent assay. Cell culture supernatants and mice sera were analyzed for cytokine content using the BD OptEIA ELISA set (BD Pharmingen) for the detection of TNF- $\alpha$, IL-6, IL-1 $\beta$, IL-12p40, IL-18 and IL-10. All assays were performed as recommended by the manufacturer.

Histology and immunohistochemistry. For immunohistochemistry of tissue sections, mouse spleens, livers, and lungs were fixed in $10 \%$ formalin and embedded in paraffin. Paraffin sections $(4 \mu \mathrm{m})$ were cut and stained with hematoxylin and eosin $(\mathrm{H} \& \mathrm{E})$. Histopathologic score was established on the basis of the numbers and distribution of inflammatory cells and the severity of inflammation within the tissues in which a board-certified pathologist independently scored each organ section without prior knowledge of the treatment groups. ${ }^{3,4}$ A histological score ranging from 0-4 was ascribed to each specimen.

Bacteria count. Blood and peritoneal lavage fluids were collected from mice by cardiac puncture at indicated time after CLP or bacterial infection. After performing serial dilution of blood, $5 \mu \mathrm{L}$ of each dilution was plated on blood agar plates. Bacteria were counted after incubation at $37^{\circ} \mathrm{C}$ for $24 \mathrm{~h}$ and calculated as counting colony-forming units per whole peritoneal lavage or blood.

In vivo imaging. A $\mathrm{WS}_{2} / \mathrm{IR} 783$ complex was prepared by simply mixing $0.5 \mathrm{mg} \mathrm{mL}^{-1}$ of $\mathrm{WS}_{2}$ nanosheets with IR783 dye $(10 \mu \mathrm{M})$ for overnight in the dark. The imaging of organs was performed using the IVIS Spectrum-CT in vivo imaging system (PerkinElmer, Inc.). A $100 \mu \mathrm{L}$ portion of $\mathrm{WS}_{2} / \mathrm{IR} 783$ complex $(8.5 \mathrm{nM})$ was injected into the tail vein or the peritoneum of a mouse. Then, the spleen, liver, lung, and kidney of the mouse were extracted and imaged at each time interval $(0.5,1,1.5,3,6,12,24,36,48$, and $72 \mathrm{~h}$ for I.V. injection and $1 \mathrm{~h}, 3 \mathrm{~h}, 12$ h, 1, 2, 3, 5 and 10 days for I.P. injection). 
Statistical analysis. All data were analyzed using Student's t-test with Bonferroni adjustment or ANOVA for multiple comparisons, and are presented as mean \pm SD. Statistical analyses were conducted using the SPSS (Version 12.0) statistical software program (SPSS, Chicago, IL, USA). Differences were considered significant at $\mathrm{p}<0.05$. For survival, data were graphed and analyzed by the product limit method of Kaplan and Meier, using the log-rank (ManteleCox) test for comparisons using GraphPad Prism (version 5.0, La Jolla, CA, USA). 


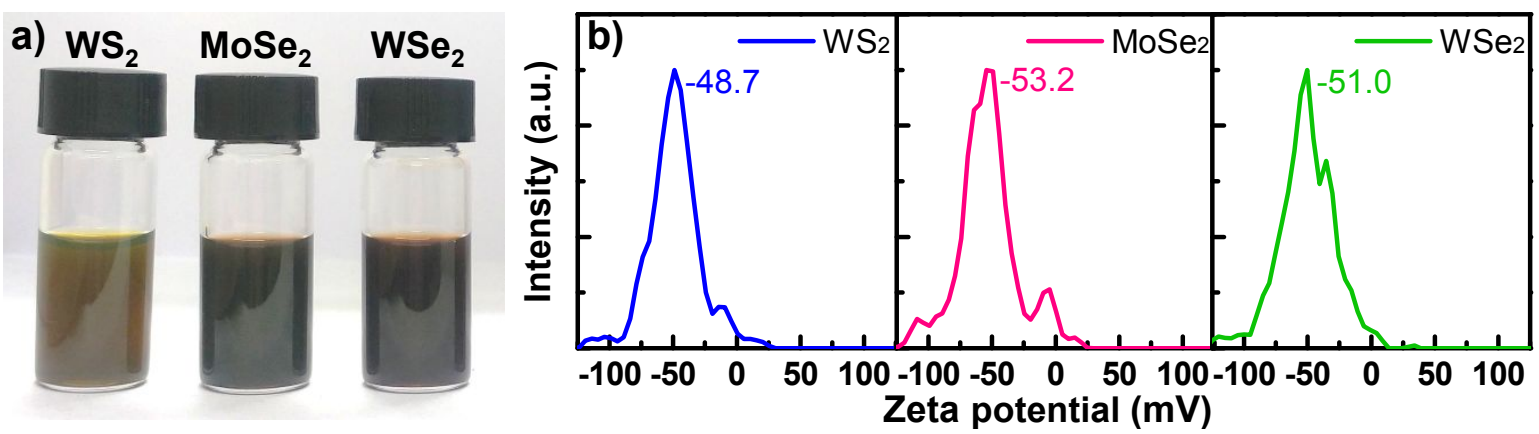

Figure S1. a) Photographs of the solutions of exfoliated TMD nanosheets. b) $\zeta$-Potentials of exfoliated $\mathrm{WS}_{2}, \mathrm{MoSe}_{2}$, and $\mathrm{WSe}_{2}$ nanosheets.

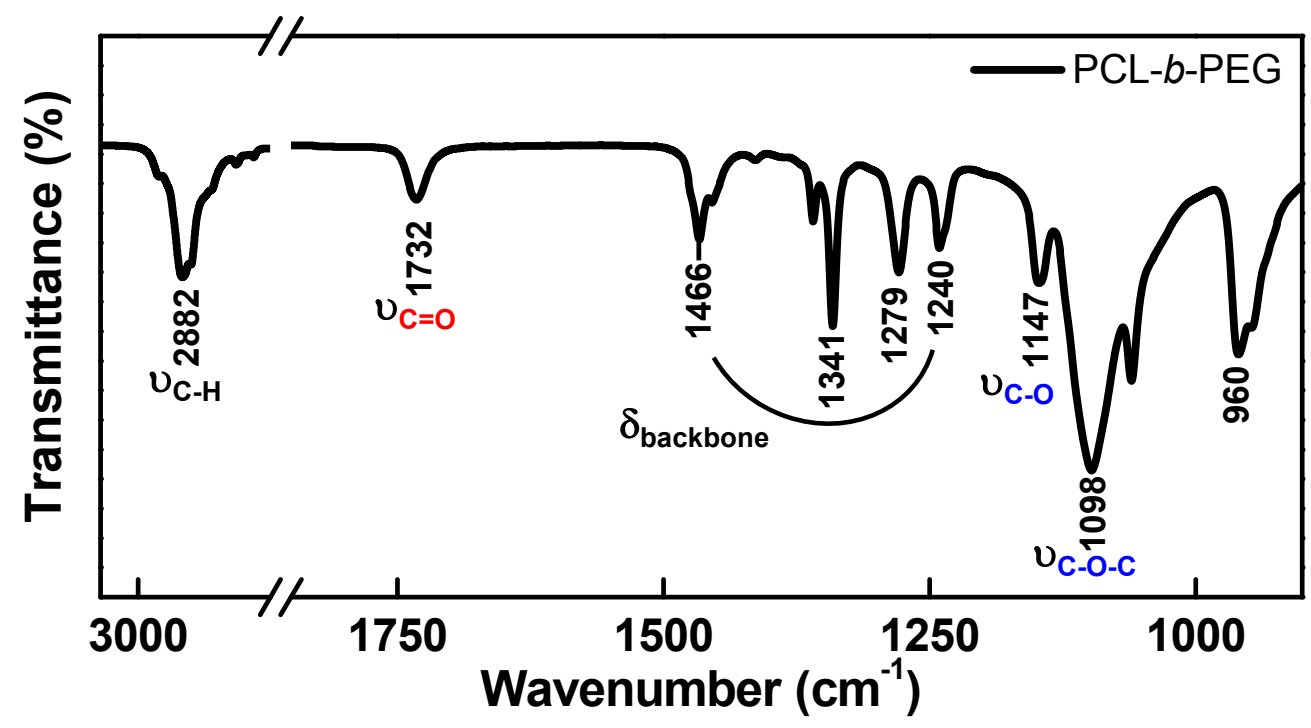

Figure S2. FT-IR spectrum of pristine PCL- $b$-PEG. 

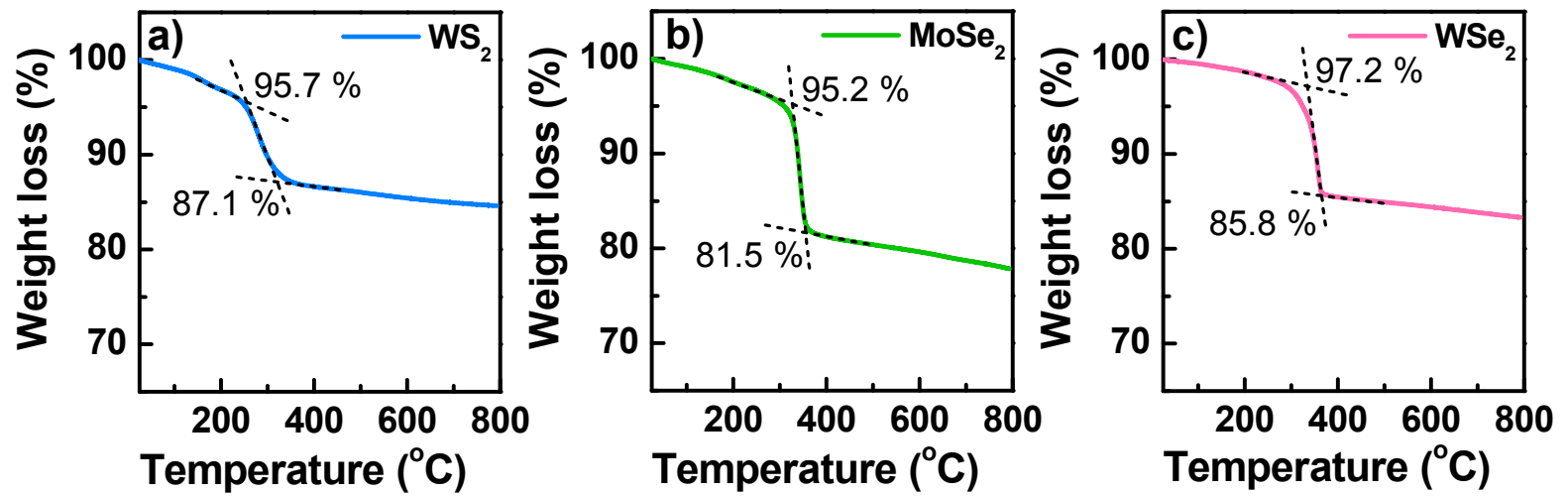

Figure S3. Thermogravimetric curves of a) $\mathrm{WS}_{2}$, b) $\mathrm{MoSe}_{2}$, and c) $\mathrm{WSe}_{2}$ nanosheets exfoliated and functionalized with PCL- $b$-PEG in water.
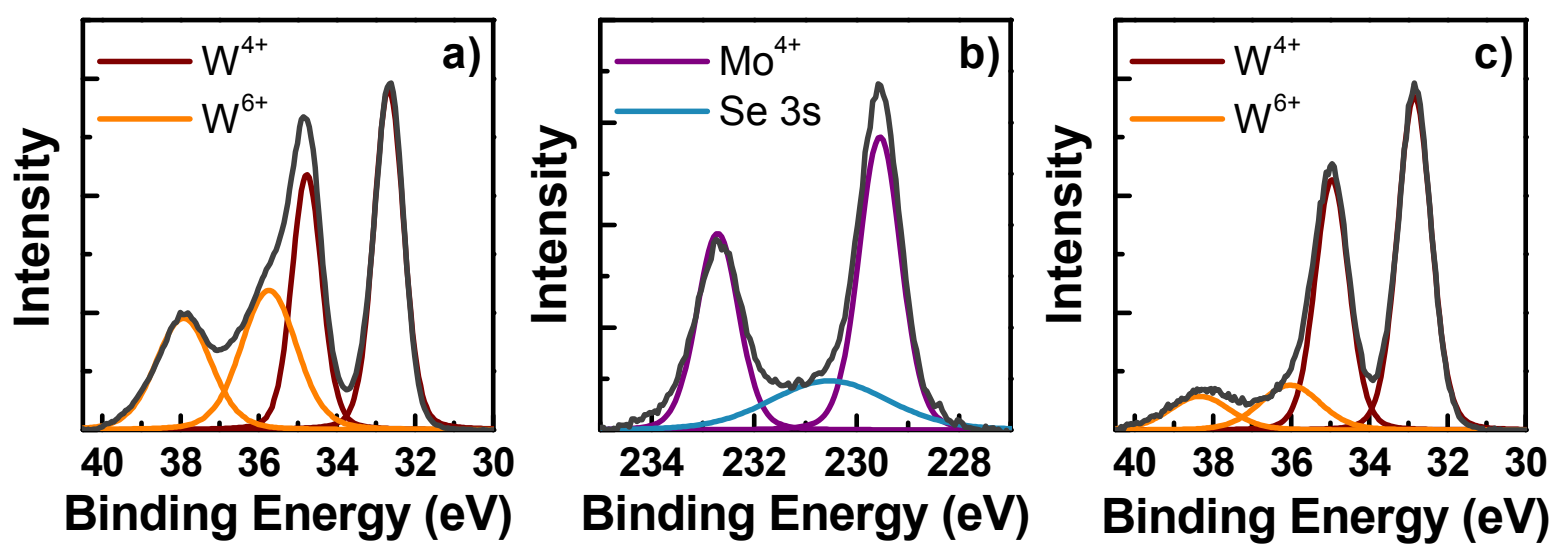

Figure S4. XPS spectra for a) W4f of $\mathrm{WS}_{2}$ nanosheets, b) Mo3d of $\mathrm{MoSe}_{2}$ nanosheets and c) W4f of $\mathrm{WSe}_{2}$ nanosheets exfoliated and functionalized with PCL- $b$-PEG in water. 

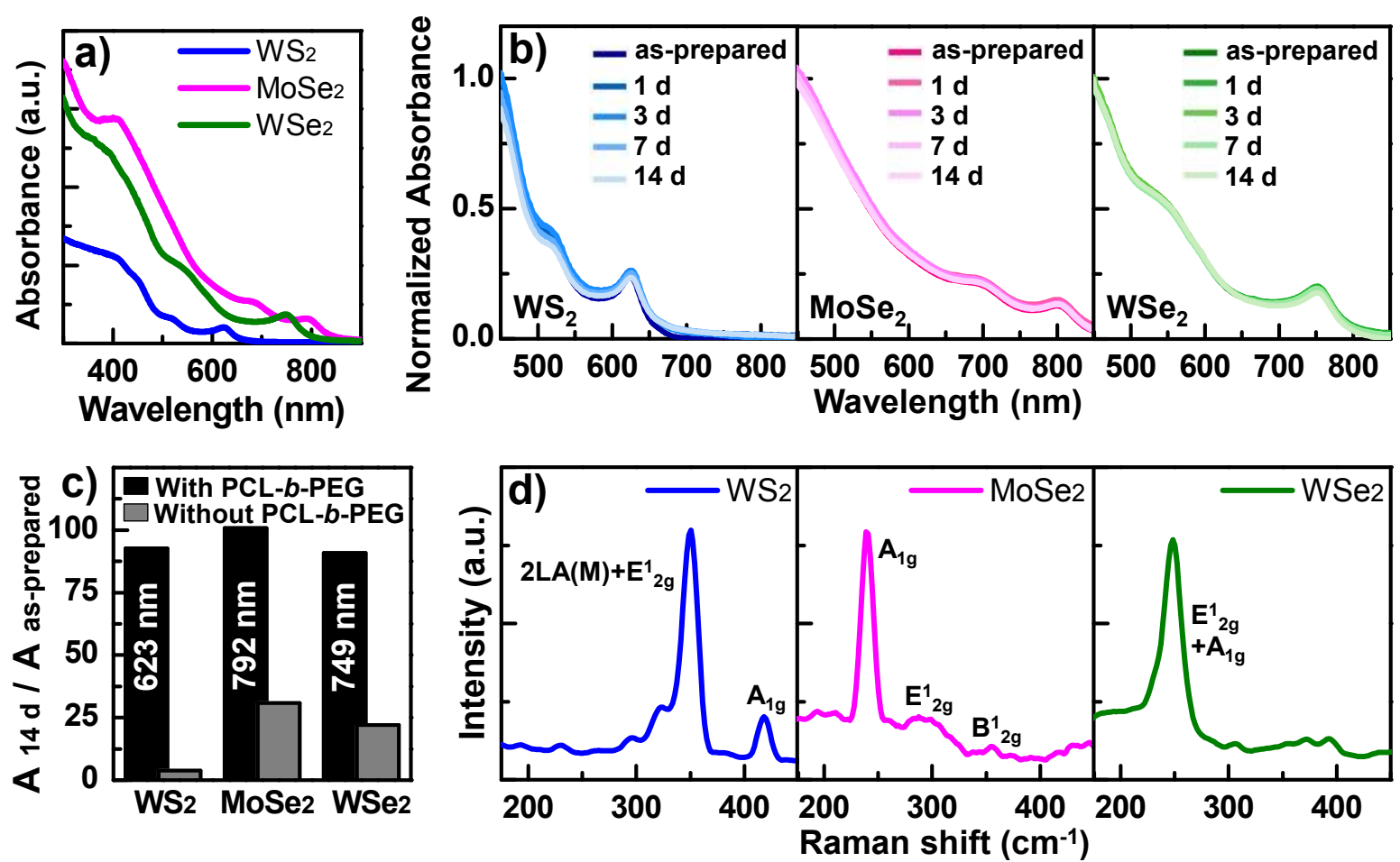

Figure S5. a) UV-Vis absorption spectra of the TMD nanosheets exfoliated by PCL- $b$-PEG.

b) Absorption profiles of the exfoliated TMD nanosheets over time in water. c) Absorbance ratios of the exfoliated TMD nanosheets standing for 14 days with the as-prepared ones at each A-excitonic wavelength. d) Raman spectra of $\mathrm{WS}_{2}, \mathrm{MoSe}_{2}$, and $\mathrm{WSe}_{2}$ nanosheets exfoliated by PCL- $b$-PEG (Excitation: $532 \mathrm{~nm}$ ). 


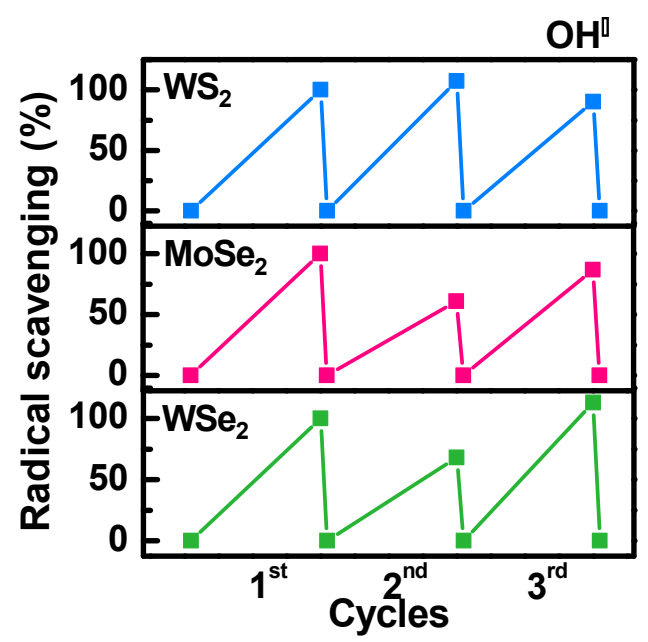

Figure S6. Sustainability of $\mathrm{WS}_{2}, \mathrm{MoSe}_{2}$, and $\mathrm{WSe}_{2}$ nanosheets exfoliated with PCL- $b$-PEG for scavenging hydroxyl radicals.

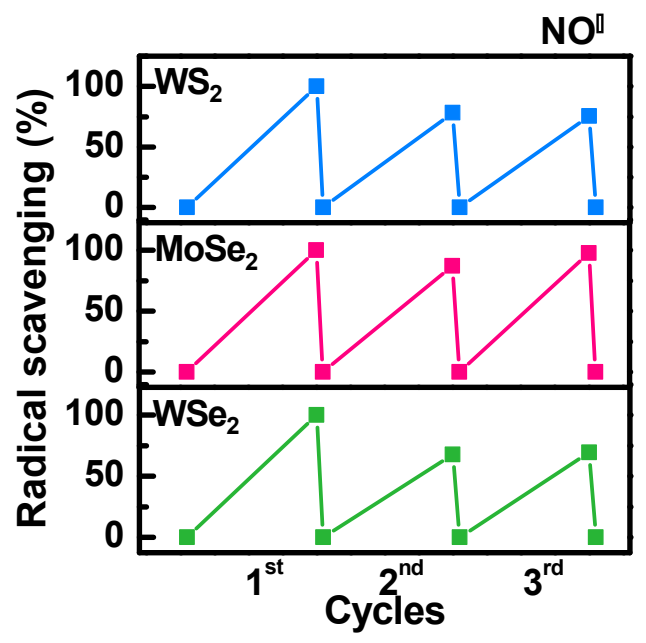

Figure S7. Sustainability of $\mathrm{WS}_{2}, \mathrm{MoSe}_{2}$, and $\mathrm{WSe}_{2}$ nanosheets exfoliated with PCL- $b$-PEG for scavenging nitric oxide. 

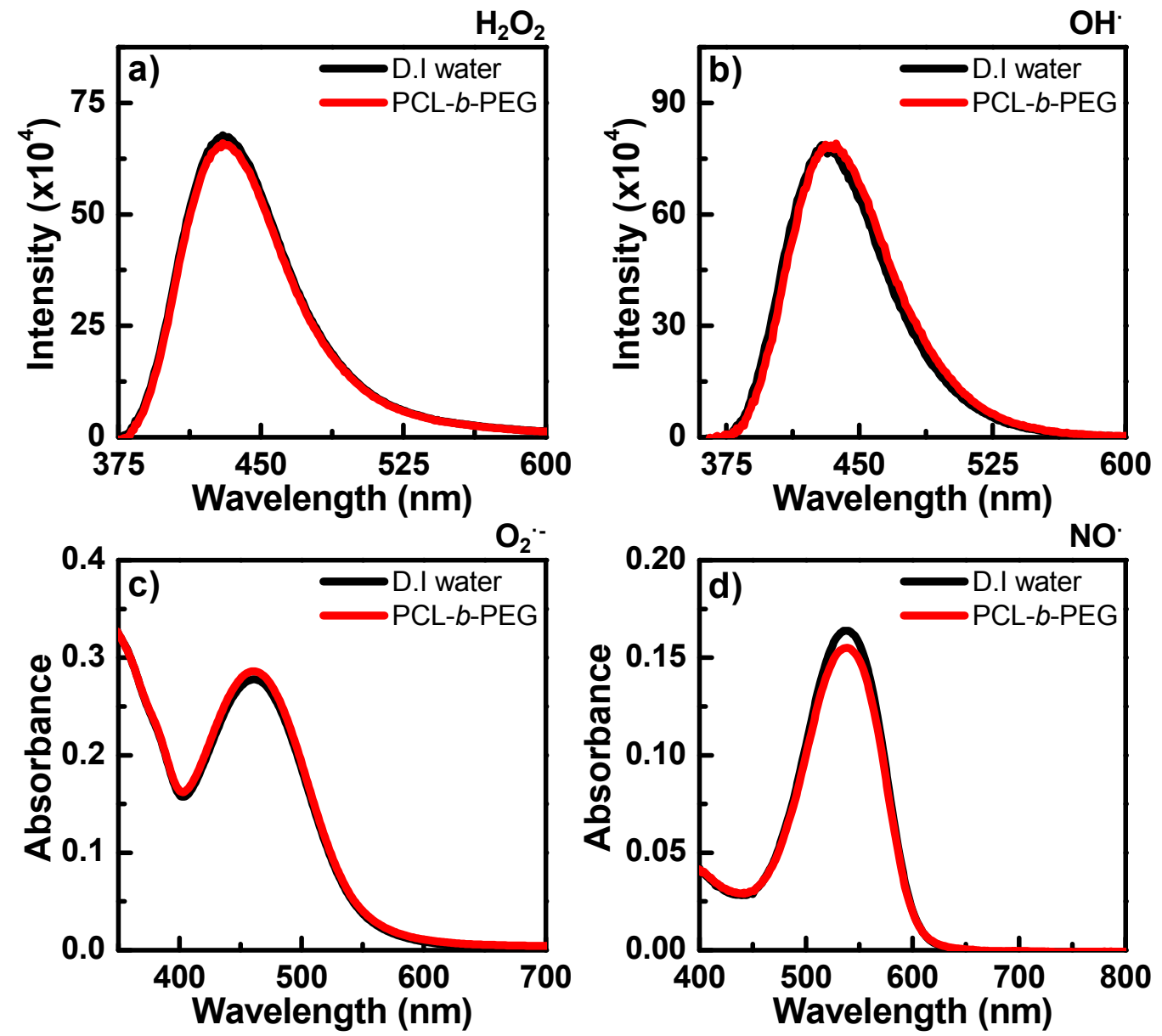

Figure S8. Scavenging activities of pristine PCL- $b$-PEG for a) Hydrogen peroxide, b) Hydroxyl radical, c) Superoxide, and d) Nitric oxide, showing no scavenging activities itself. 

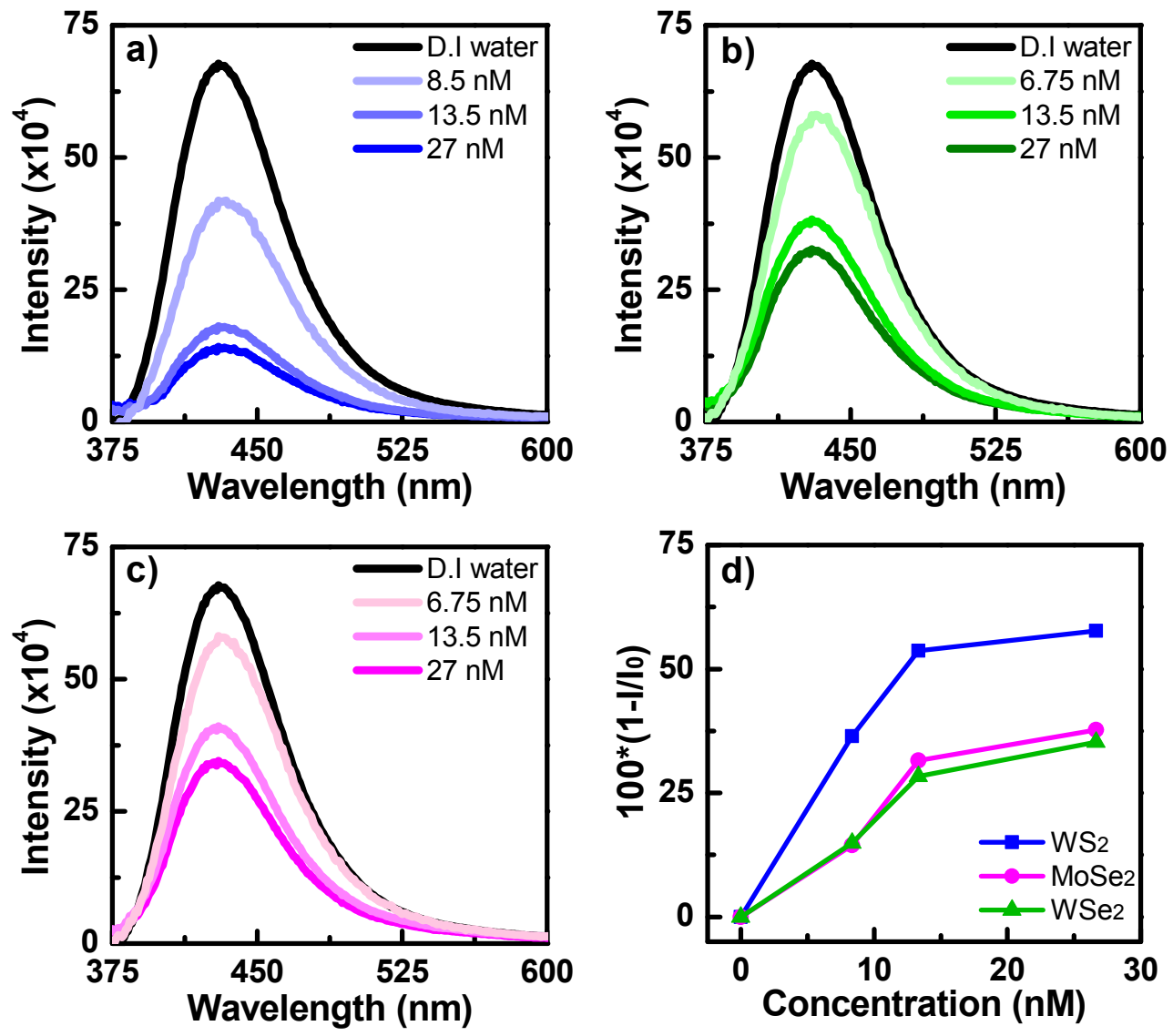

Figure S9. $\mathrm{H}_{2} \mathrm{O}_{2}$ scavenging activities of a) $\mathrm{WS}_{2}$, b) $\mathrm{MoSe}_{2}$, and c) $\mathrm{WSe}_{2}$ nanosheets at various concentrations. d) Plots of TMD concentration-dependent scavenging activities. 

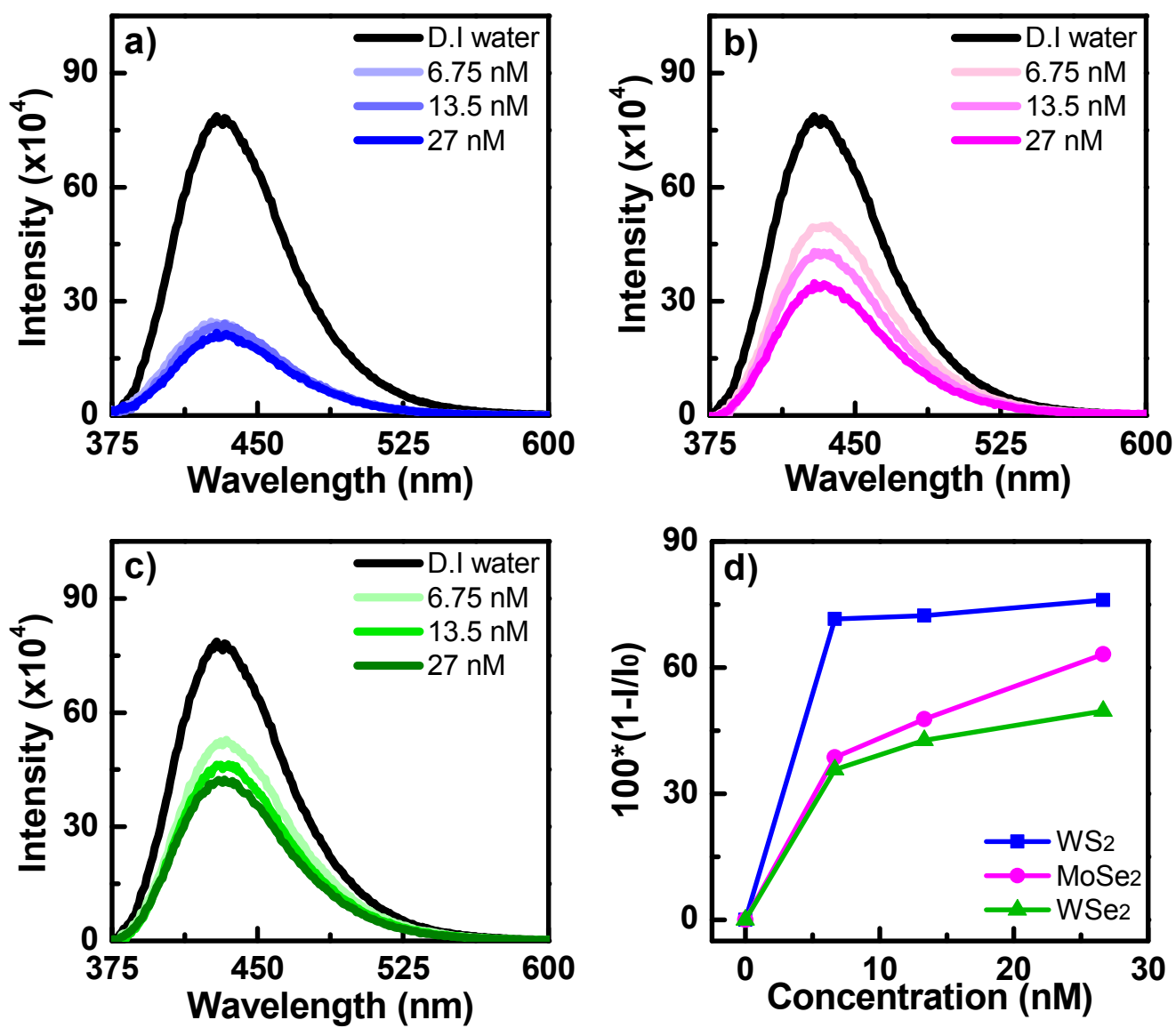

Figure S10. Hydroxyl radical scavenging activities of a) $\mathrm{WS}_{2}$, b) $\mathrm{MoSe}_{2}$, and c) $\mathrm{WSe}_{2}$ nanosheets at various concentrations. d) Plots of TMD concentration-dependent scavenging activities. 

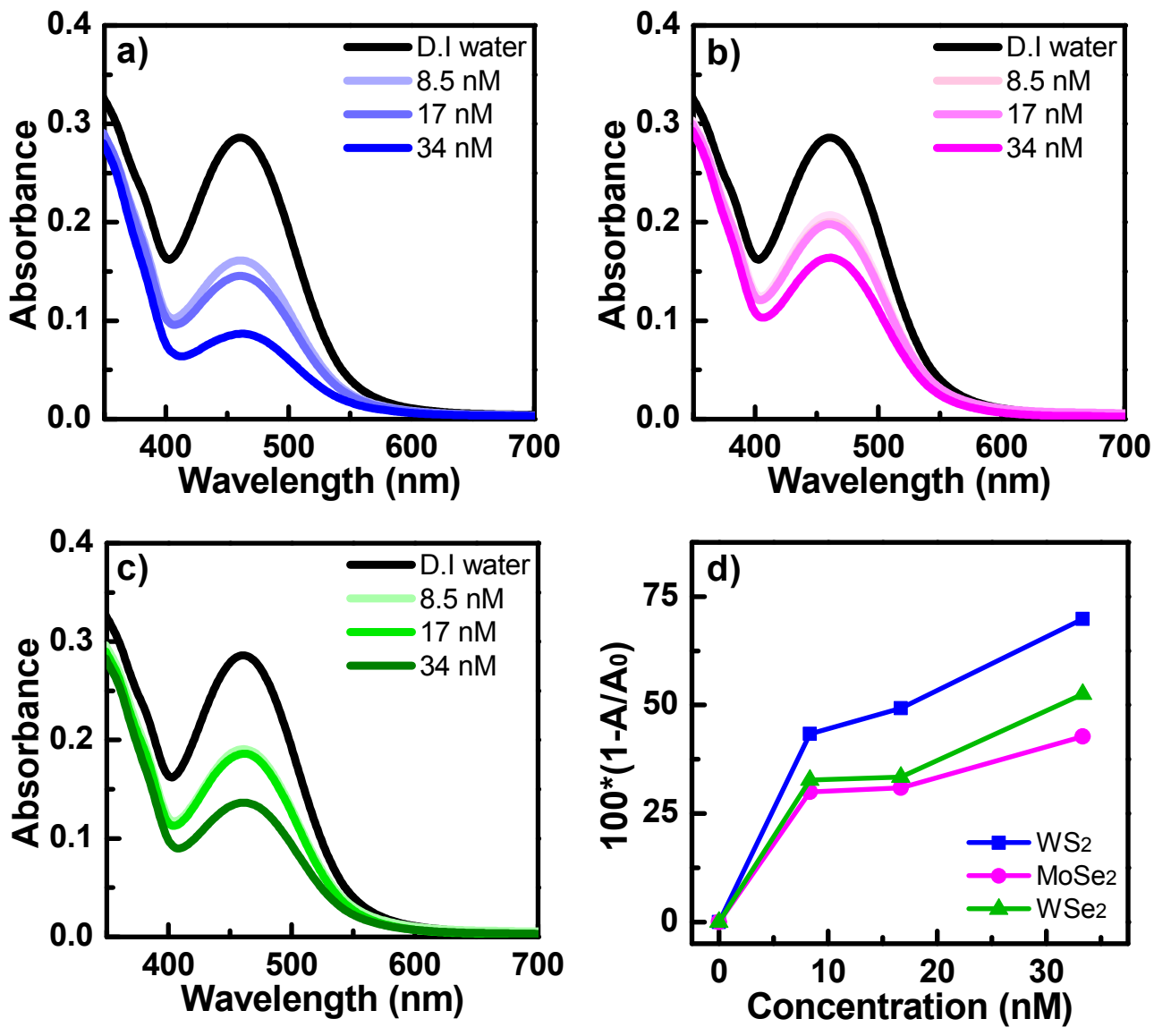

Figure S11. Superoxide scavenging activities of a) $\mathrm{WS}_{2}$, b) $\mathrm{MoSe}_{2}$, and c) $\mathrm{WSe}_{2}$ nanosheets at various concentrations. d) Plots of TMD concentration-dependent scavenging activities. 

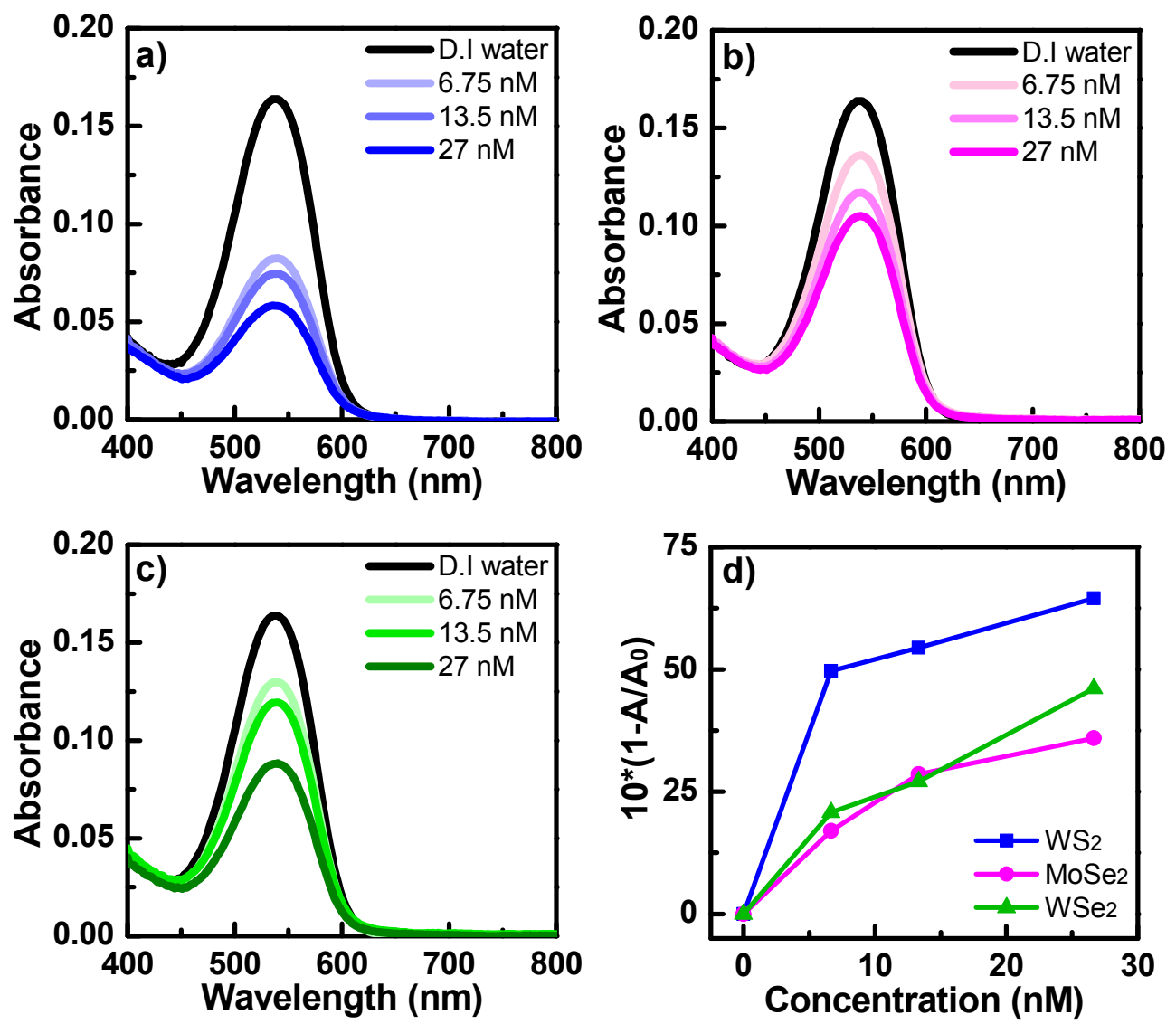

Figure S12. Nitric oxide scavenging activities of a) $\mathrm{WS}_{2}$, b) $\mathrm{MoSe}_{2}$, and c) $\mathrm{WSe}_{2}$ nanosheets at various concentrations. d) Plots of TMD concentration-dependent scavenging activities. 

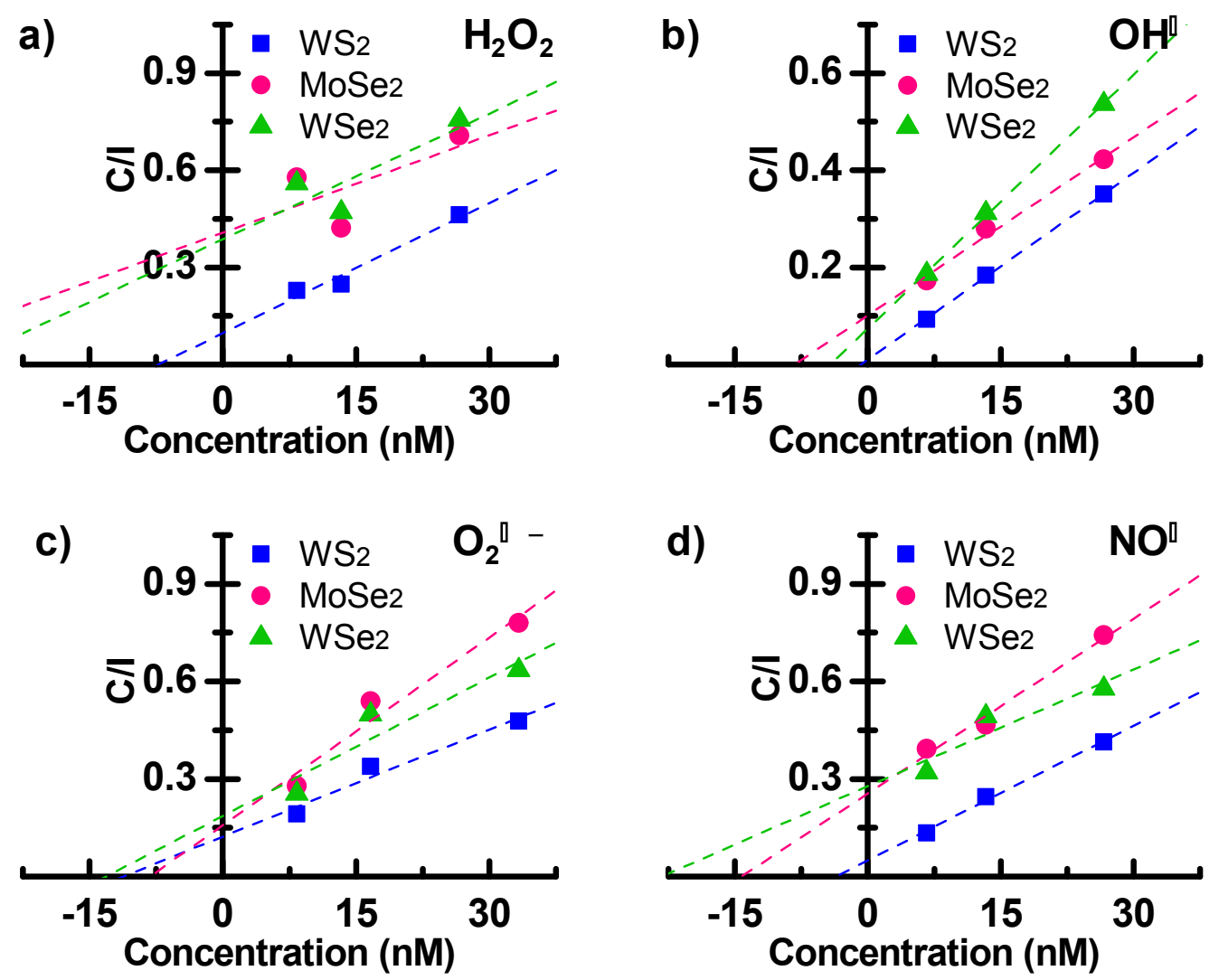

Figure S13. Langmuir-isotherm plots for a) $\mathrm{H}_{2} \mathrm{O}_{2}$ scavenging activities, b) Hydroxyl radical scavenging activities, c) Superoxide scavenging activities, and d) Nitric oxide scavenging activities of $\mathrm{WS}_{2}, \mathrm{MoSe}_{2}$, and $\mathrm{WSe}_{2}$ nanosheets at various concentrations to extract the equilibrium constants of scavenging $\left(K_{\mathrm{s}}\right)$. 

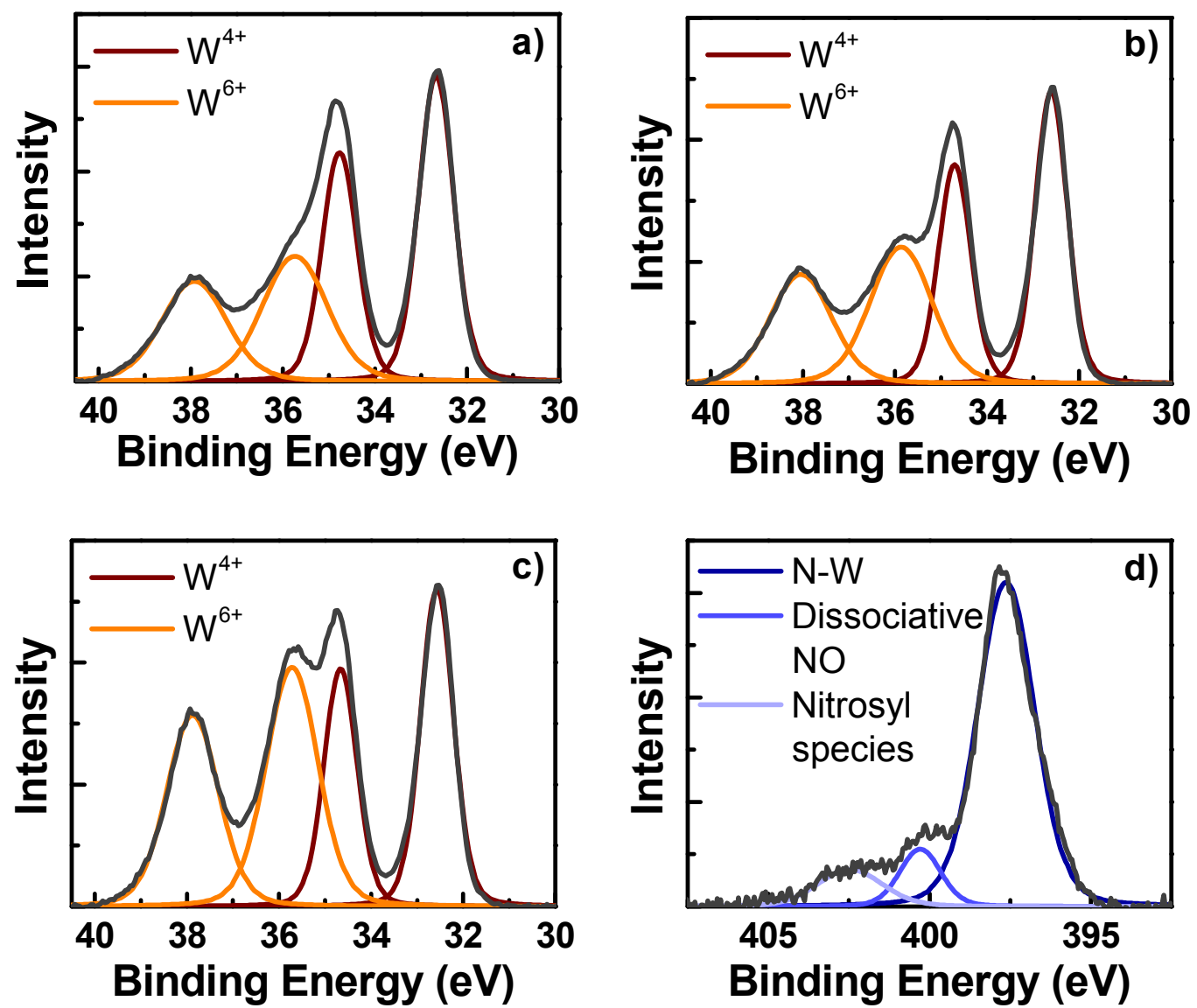

Figure S14. XPS spectra of $\mathrm{WS}_{2}$ nanosheets after scavenging ROS and RNS. W4f XPS spectra of a) As-prepared $\mathrm{WS}_{2}$ nanosheets, and b) The $\mathrm{WS}_{2}$ after scavenging hydrogen peroxide and c) The $\mathrm{WS}_{2}$ after scavenging nitric oxide. d) N1s XPS spectrum of the $\mathrm{WS}_{2}$ nanosheets after scavenging nitric oxide. 
a) $\mathrm{MX}_{2}+\cdot \mathrm{ROS}+\mathrm{H}_{2} \mathrm{O} \longrightarrow \mathrm{HO}-\mathrm{MX}_{2}+$ Red-ROS

$$
\begin{aligned}
& \text { HO-MX }{ }_{2}+\cdot \text { ROS }+\mathrm{H}_{2} \mathrm{O} \longrightarrow \mathrm{XV}-\mathrm{MX}+\text { Red-ROS } \\
& \mathrm{XV}-\mathrm{MX}+\cdot \mathrm{ROS}+\mathrm{H}_{2} \mathrm{O} \longrightarrow \mathrm{O}-\mathrm{MX}+\text { Red-ROS }
\end{aligned}
$$

b) $2 \mathrm{XV}-\mathrm{MX}+2 \mathrm{NO} \longrightarrow 2 \mathrm{ON}-\mathrm{MX}$

$$
\begin{aligned}
& \mathrm{ON}-\mathrm{MX}+\mathrm{NO}^{\circ} \longrightarrow \mathrm{N}-\mathrm{MX}+\mathrm{NO}_{2} \\
& \mathrm{XV-MX}+\mathrm{NO}_{2} \longrightarrow \mathrm{O}-\mathrm{MX}+\mathrm{NO}^{\circ} \\
& \mathrm{XV-MX}+\mathrm{NO}^{\circ} \longrightarrow \mathrm{ON}-\mathrm{MX}
\end{aligned}
$$

Figure S15. Proposed mechanisms responsible for scavenging a) ROS and b) RNS by $2 \mathrm{H}-$ TMD nanosheets. M (transition metal atom), $\mathrm{X}$ (chalcogen atom), HO-MX 2 (hydroxylated TMD), XV-MX (chalcogen-vacant TMD), Red-ROS (reduced ROS), O-MX (oxidized TMD), ON-MX (nitrosylated TMD) and N-MX (N-bearing TMD). 

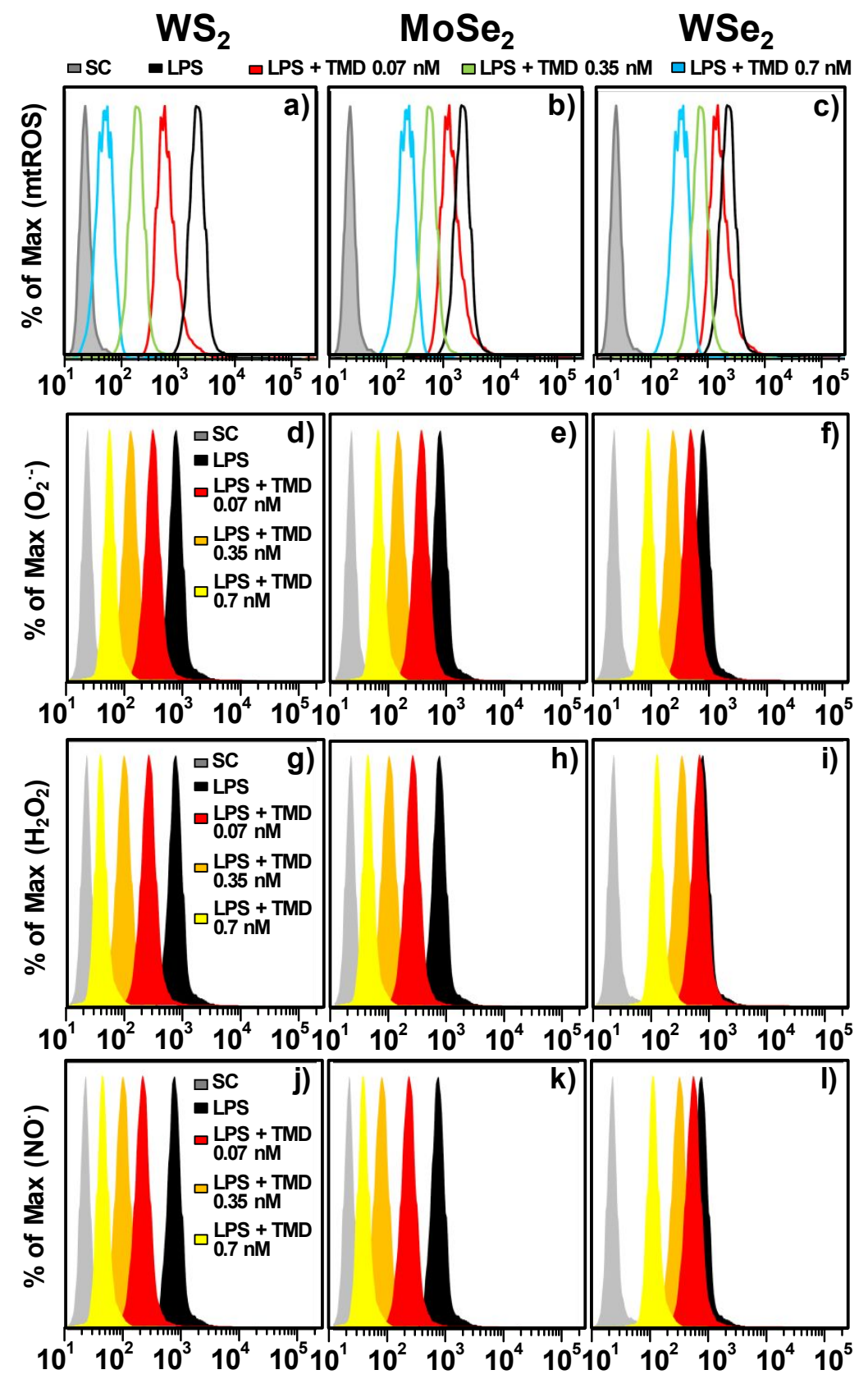

Figure S16. ROS and RNS scavenging activities of TMD nanosheets in LPS-induced inflammatory BMDMs. a)-c) Mitochondrial ROS scavenging activities of $\mathrm{WS}_{2}, \mathrm{MoSe}_{2}$, and $\mathrm{WSe}_{2}$ nanosheets determined by FACS. d)-f) Intracellular superoxide scavenging activities, g)i) Intracellular $\mathrm{H}_{2} \mathrm{O}_{2}$ scavenging activities, and j)-1) Intracellular nitric oxide scavenging activities of $\mathrm{WS}_{2}, \mathrm{MoSe}_{2}$, and $\mathrm{WSe}_{2}$ nanosheets. BMDMs were stimulated with LPS for 30 min, followed by treatment with TMDs for $45 \mathrm{~min}$. 


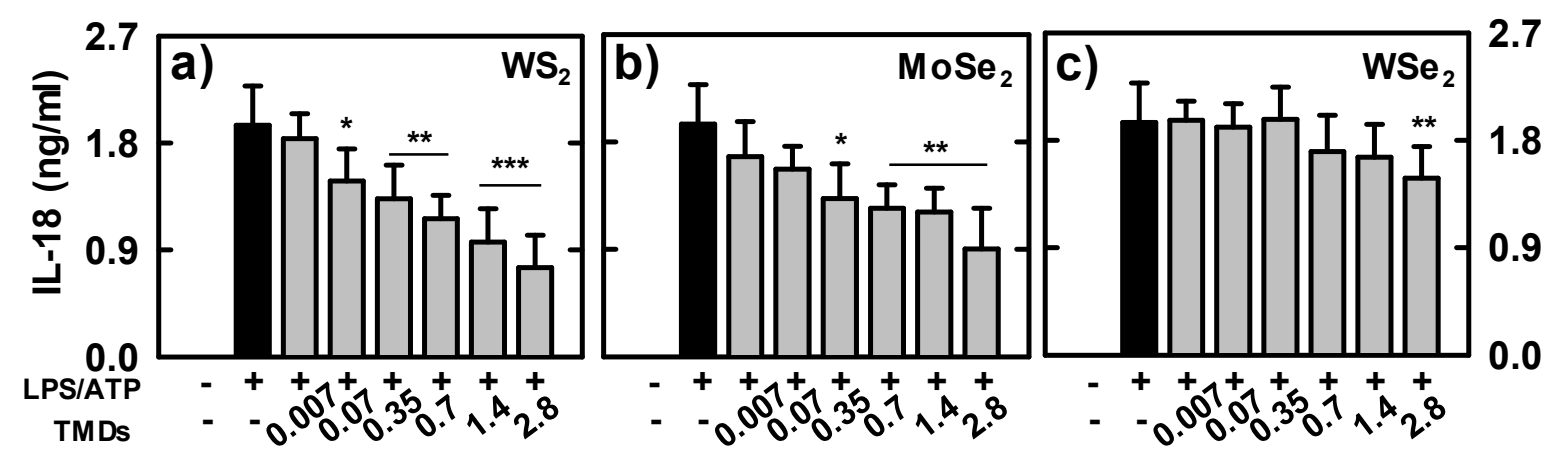

Figure S17. Effects of TMD nanosheets on acute-phase inflammatory cytokine IL-18 in BMDMs stimulated with LPS/ATP for 1 h. a) $\mathrm{WS}_{2}$, b) $\mathrm{MoSe}_{2}$, and c) $\mathrm{WSe}_{2}$ nanosheets. Culture supernatants were harvested and analyzed by ELISA. Data are the means \pm SD of three experiments. Significant differences $(* p<0.01 ; * * p<0.05 ; * * * p<0.001)$ compared with $\mathrm{LPS} / \mathrm{ATP}+\mathrm{SC}$.

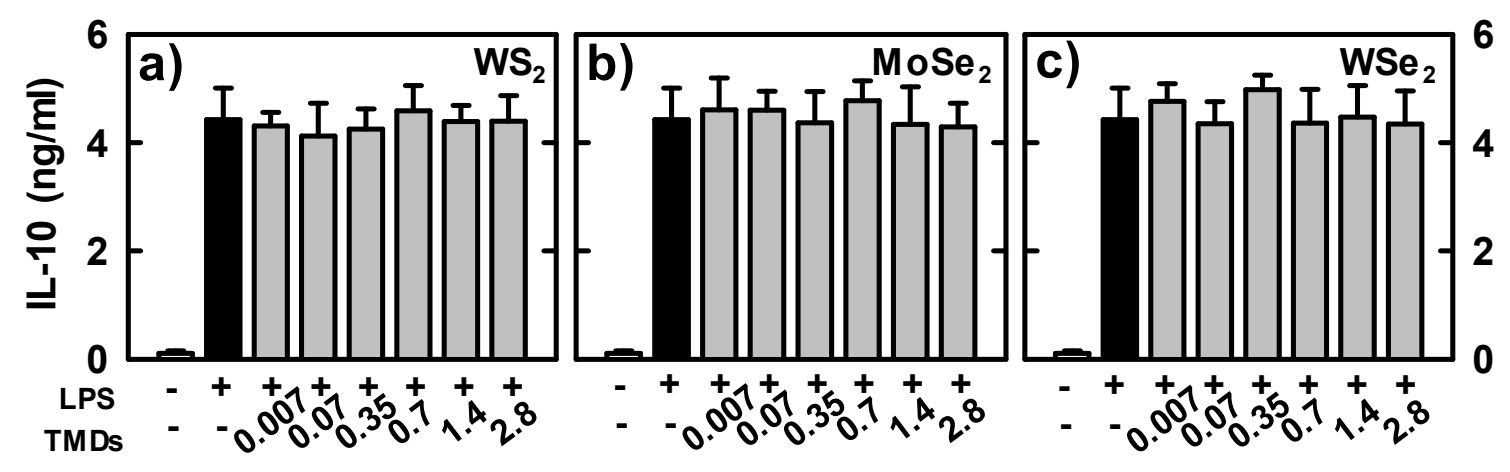

Figure S18. Effects of TMD nanosheets on the secretion levels of anti-inflammatory cytokine IL-10 in BMDMs stimulated with LPS for $18 \mathrm{~h}$. a) $\mathrm{WS}_{2}$, b) $\mathrm{MoSe}_{2}$, and c) $\mathrm{WSe}_{2}$ nanosheets. Culture supernatants were harvested and analyzed by ELISA. Data are the means \pm SD of three experiments, showing no significant differences compared with LPS $+\mathrm{SC}$. 

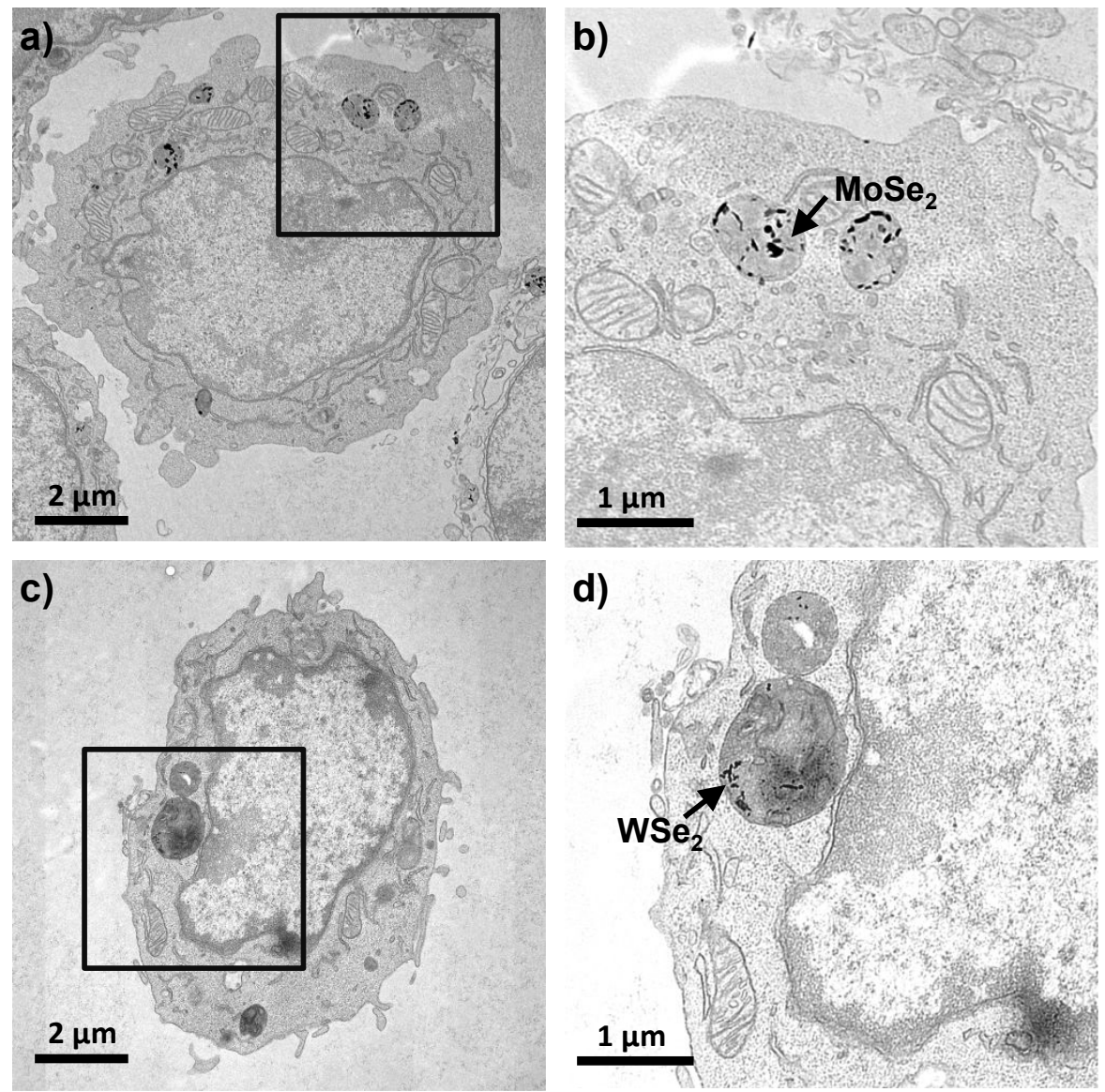

Figure S19. Endocytosis of TMD nanosheets into RAW 264.7 cells. HVEM images of a)-b) $\mathrm{MoSe}_{2}$ nanosheets-treated cells and c)-d) $\mathrm{WSe}_{2}$ nanosheets-treated cells. 


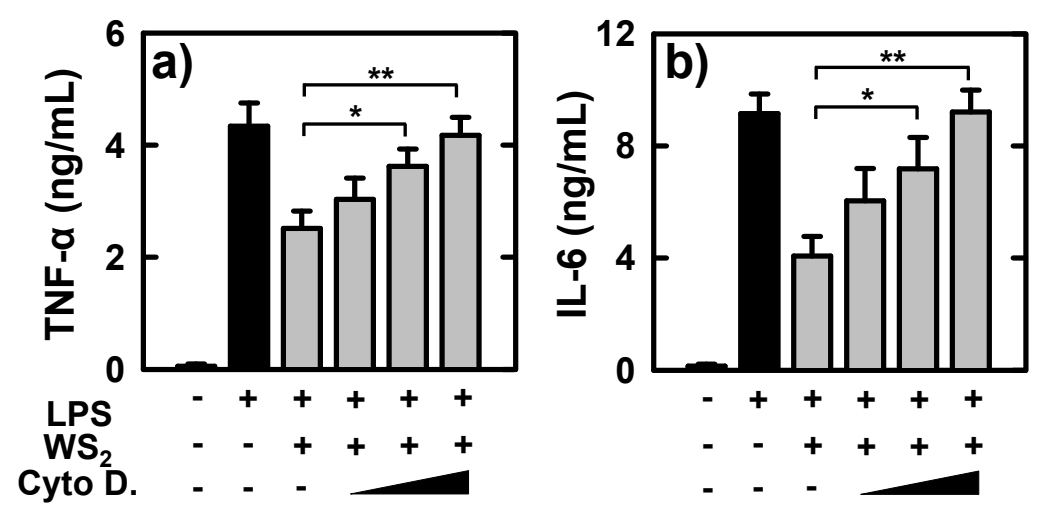

Figure S20. Effects of the endocytosis of TMD nanosheets on the secretion levels of inflammatory cytokines. Secretion levels of a) TNF- $\alpha$ and b) IL-6 in Raw264.7 cells preincubated with cytochalasin D (Cyto D, 5, 10, and $20 \mu \mathrm{M})$, an endocytosis inhibitor, for 30 min before treatment with $\mathrm{WS}_{2}$ nanosheets for $45 \mathrm{~min}$. The cells were then stimulated with LPS for $18 \mathrm{~h}$, and the supernatants were analyzed by ELISA. Data are the means \pm SD of three experiments. Significant differences $(* \mathrm{p}<0.01 ; * * \mathrm{p}<0.05 ; * * * \mathrm{p}<0.001)$ compared with LPS+SC. 

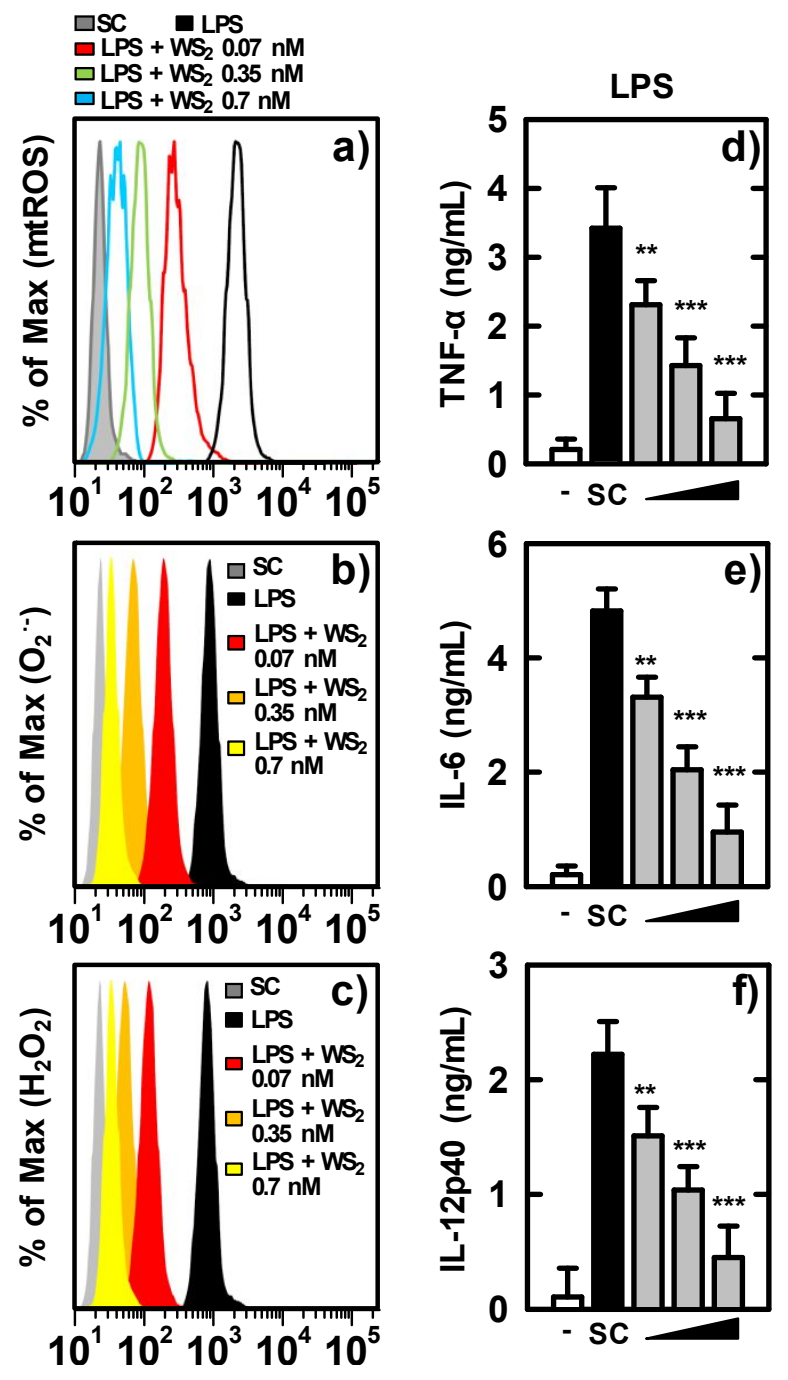

Figure S21. Activities of $\mathrm{WS}_{2}$ nanosheets on scavenging ROS and suppression of inflammatory cytokines in human monocytes. a) Mitochondrial ROS scavenging activities of $\mathrm{WS}_{2}$ nanosheets at various concentrations measured by FACS. b) Intracellular superoxide scavenging activities and c) Intracellular $\mathrm{H}_{2} \mathrm{O}_{2}$ scavenging activities of $\mathrm{WS}_{2}$ nanosheets at various concentrations in human monocytes stimulated with LPS for $30 \mathrm{~min}$. Secretion levels of d) TNF- $\alpha$, e) IL-6, and IL-12p40 in the culture supernatants of the human monocytes stimulated with LPS for $18 \mathrm{~h}$ after pretreatment with $\mathrm{WS}_{2}$ nanosheets for $45 \mathrm{~min}$. Culture supernatants were harvested and analyzed by ELISA. Results are expressed as means \pm SD of three experiments. Significant differences $(* p<0.01 ; * * p<0.05 ; * * * p<0.001)$ compared with LPS+SC. 
a)
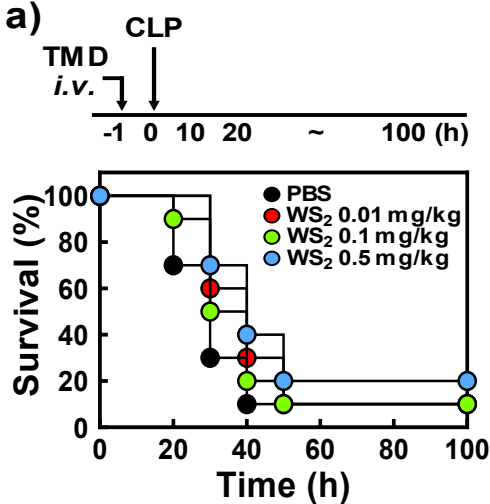

b)
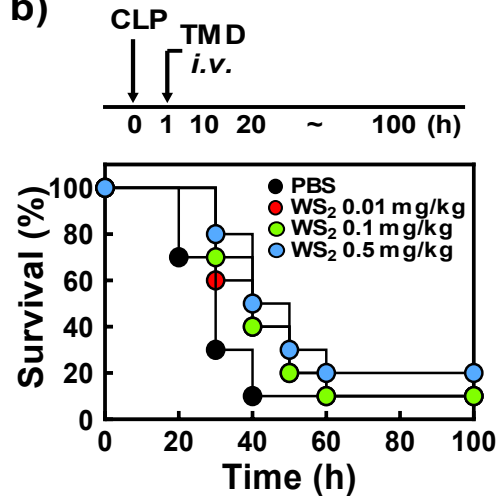

Figure S22. Therapeutic efficacy of $\mathrm{WS}_{2}$ nanosheets on CLP-induced bacteremia mice. a) Survival rates of the septic mice intravenously (i.v.) pre-administrated with $\mathrm{WS}_{2}$ nanosheets before the CLP procedure. b) Survival rates of the septic mice intravenously administrated with $\mathrm{WS}_{2}$ nanosheets $1 \mathrm{~h}$ after the CLP procedure ( $\mathrm{n}=10$ mice per group).
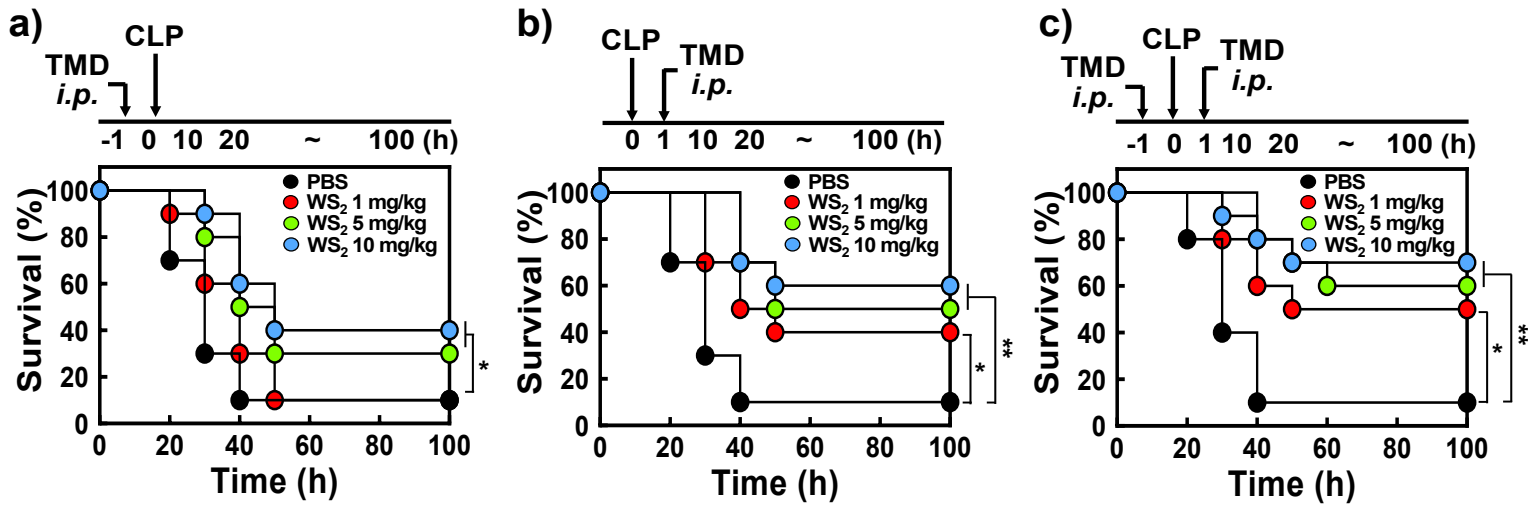

Figure S23. Therapeutic efficacy of $\mathrm{WS}_{2}$ nanosheets on CLP-induced bacteremia mice. a) Survival rates of the septic mice intraperitoneally (i.p.) pre-administrated with $\mathrm{WS}_{2}$ nanosheets before the CLP procedure. b) Survival rates of the septic mice intraperitoneally administrated with $\mathrm{WS}_{2}$ nanosheets $1 \mathrm{~h}$ after the CLP procedure. c) Survival rates of the septic mice intraperitoneally administrated with $\mathrm{WS}_{2}$ nanosheets $1 \mathrm{~h}$ before and after the CLP procedure ( $\mathrm{n}=10$ mice per group). Statistical differences compared to the CLP+PBS mice are indicated (log-rank test). 


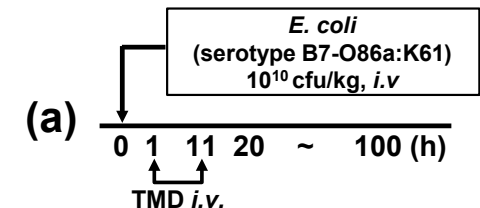

TMD i.v.

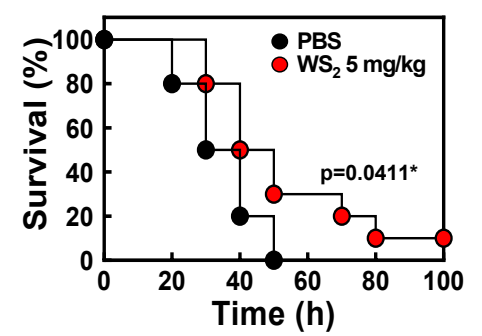

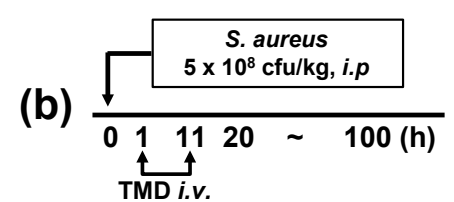
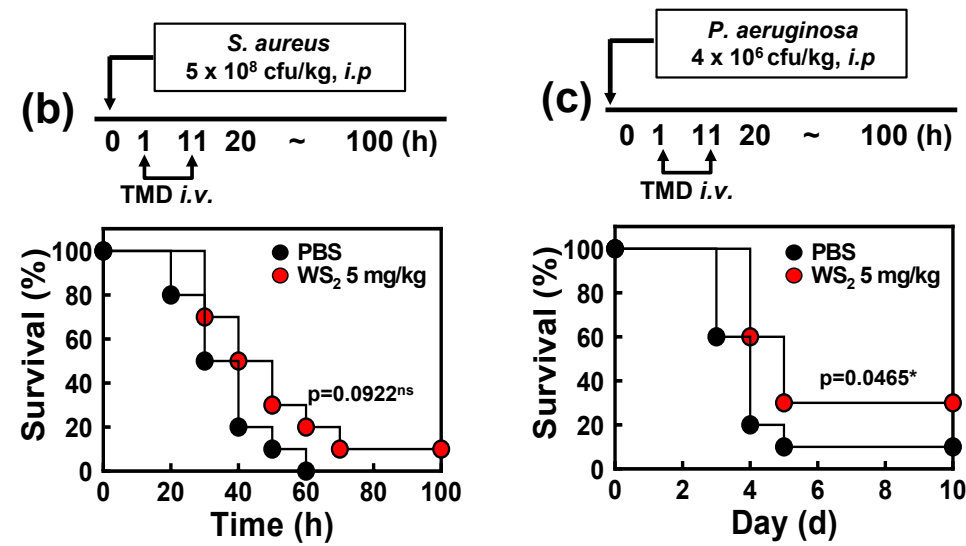

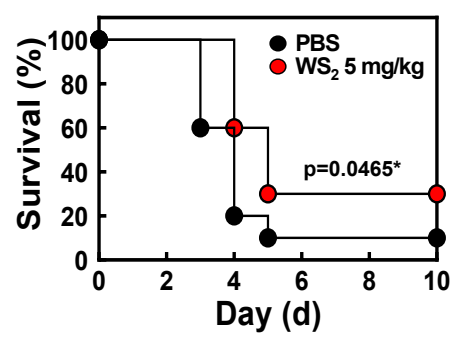

Figure S24. Therapeutic efficacy of intravenously injected $\mathrm{WS}_{2}$ nanosheets on CLP-induced polymicrobial septic mice. Survival rates of the CLP septic mice intravenously (i.v.) administrated with the $\mathrm{WS}_{2}$ nanosheets at 1 and $11 \mathrm{~h}$ after a) E. coli infection, b) S. aureus infection, and c) P. aeruginosa infection. Significant differences (ns: none significant; ${ }^{*} \mathrm{p}<$ $0.01 ; * * \mathrm{p}<0.05 ; * * * \mathrm{p}<0.001)$ compared with bacteria-infected mice not treated with $\mathrm{WS}_{2}$ nanosheets. 

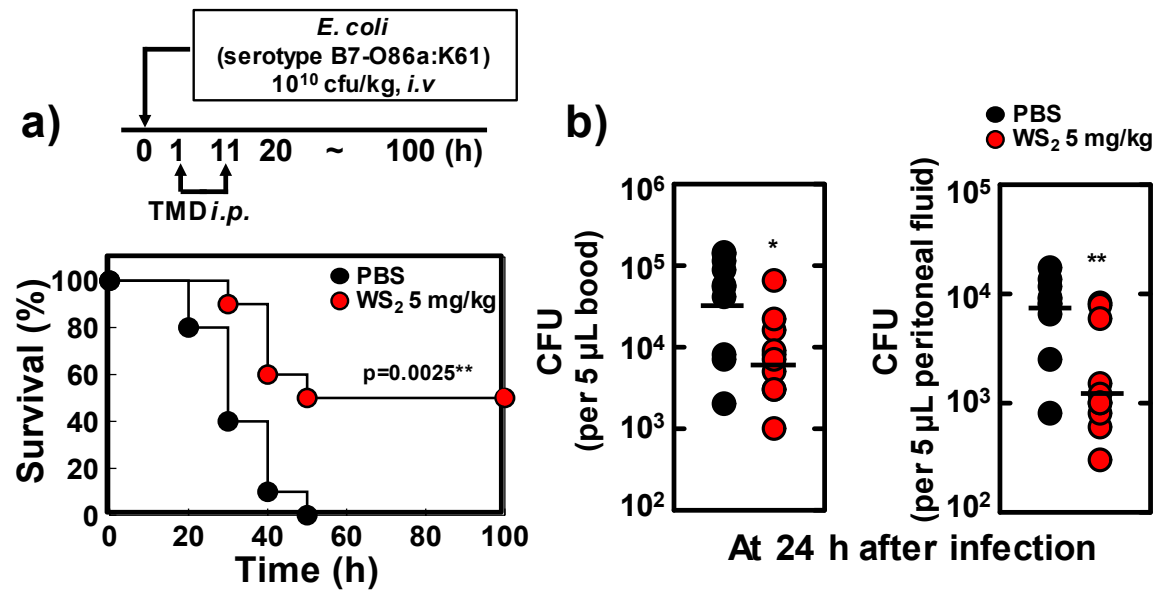

At $24 \mathrm{~h}$ after infection

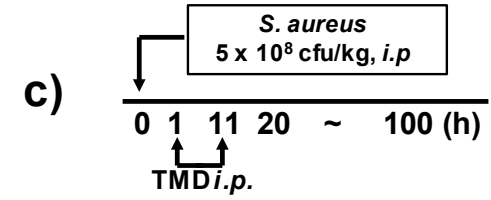

d)

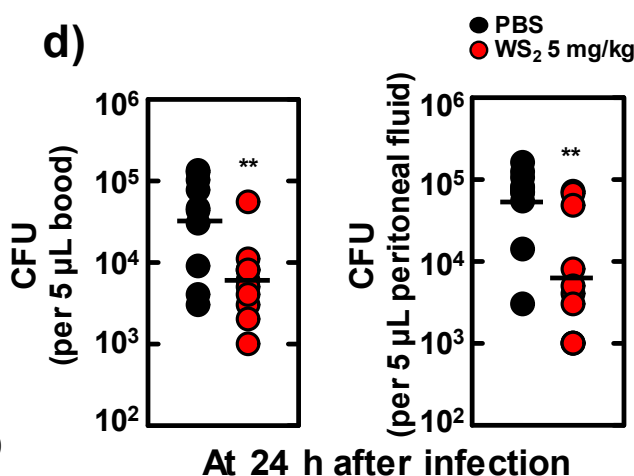

At $24 \mathrm{~h}$ after infection

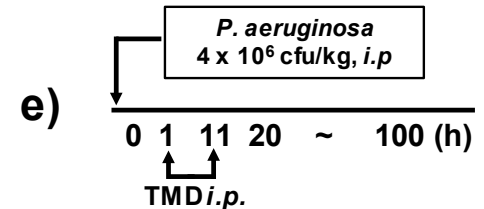

f)

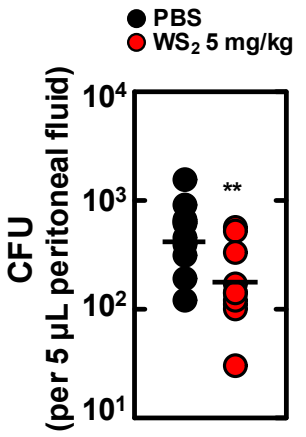

At $2 \mathrm{~d}$ after infection

Figure S25. Therapeutic efficacy of $\mathrm{WS}_{2}$ nanosheets on CLP-induced polymicrobial septic mice. a) Survival rates of the CLP septic mice intraperitoneally (i.p.) administrated with $\mathrm{WS}_{2}$ nanosheet at 1 and $11 \mathrm{~h}$ after $E$. coli infection. b) E. coli colony counts in blood and peritoneal fluid $24 \mathrm{~h}$ and 2 days after infection. c) Survival rates of the CLP septic mice intraperitoneally administrated with $\mathrm{WS}_{2}$ nanosheet at 1 and $11 \mathrm{~h}$ after $S$. aureus infection. d) S. aureus colony counts in blood and peritoneal fluid $24 \mathrm{~h}$ and 2 days after infection. e) Survival rates of the 
CLP septic mice intraperitoneally administrated with $\mathrm{WS}_{2}$ nanosheet at 1 and $11 \mathrm{~h}$ after $P$. aeruginosa infection. f) $P$. aeruginosa colony counts in blood and peritoneal fluid $24 \mathrm{~h}$ and 2 days after infection. Mortality was measured for $n=10$ mice per group (lower). Statistical differences compared to the bacteria-infected mice are indicated (log-rank test). Significant differences $\left({ }^{*} \mathrm{p}<0.01 ;{ }^{*} \mathrm{p}<0.05 ; * * * \mathrm{p}<0.001\right)$ compared with bacteria-infected mice not treated with $\mathrm{WS}_{2}$ nanosheets.

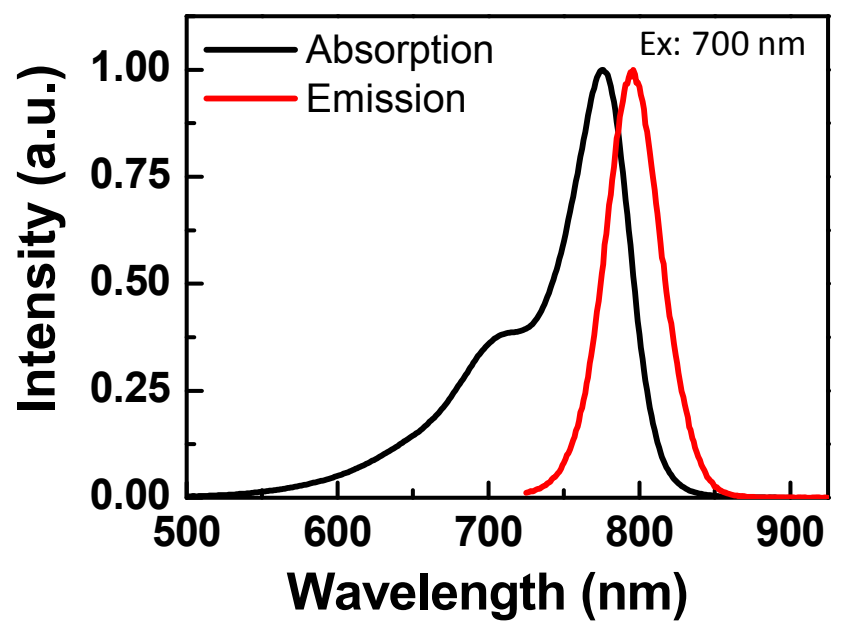

Figure S26. UV-Vis absorption and fluorescence spectra of IR783. 

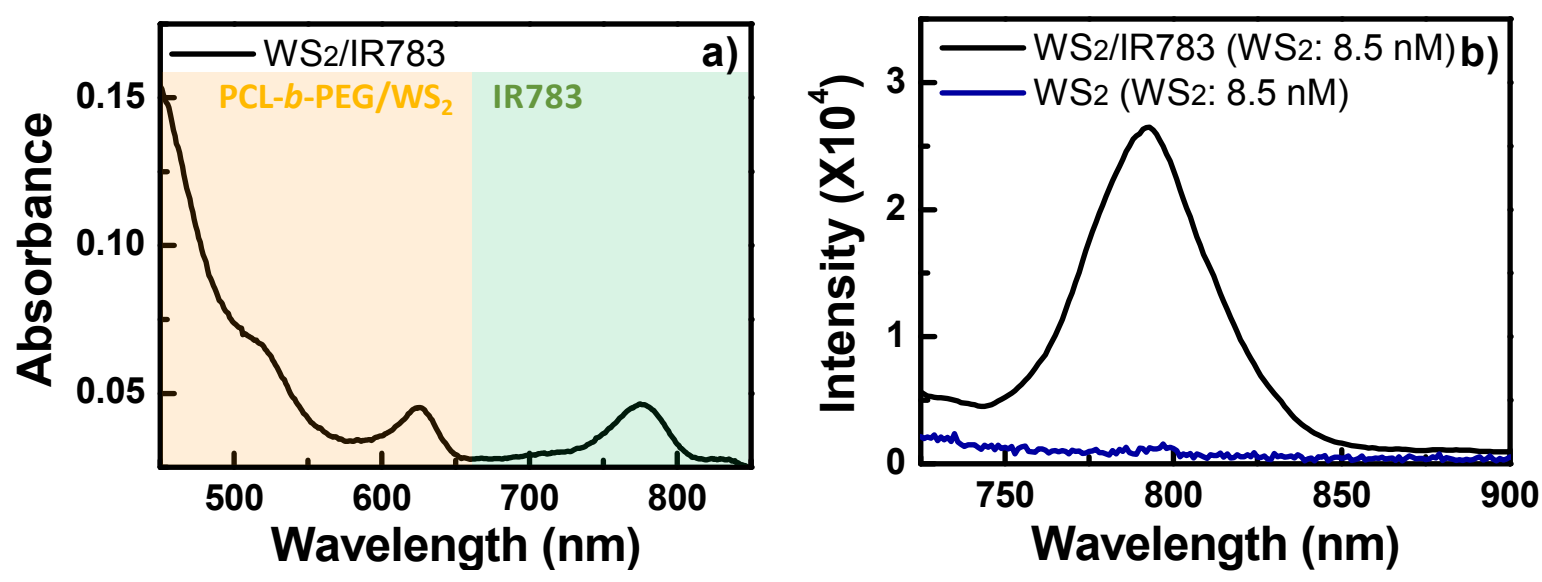

Figure S27. a) UV-Vis absorption spectrum and b) Fluorescence spectrum of $\mathrm{WS}_{2} / \mathrm{IR} 783$.

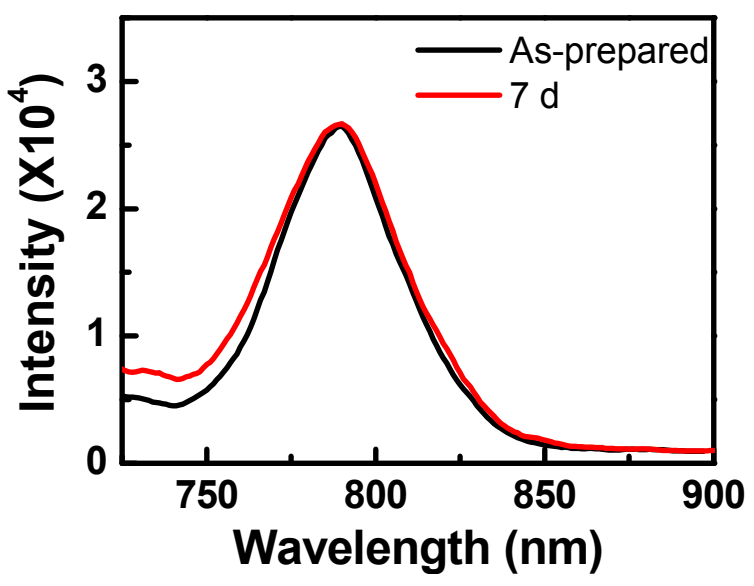

Figure S28. Fluorescence stability of $\mathrm{WS}_{2} / \mathrm{IR} 783$ over time. 

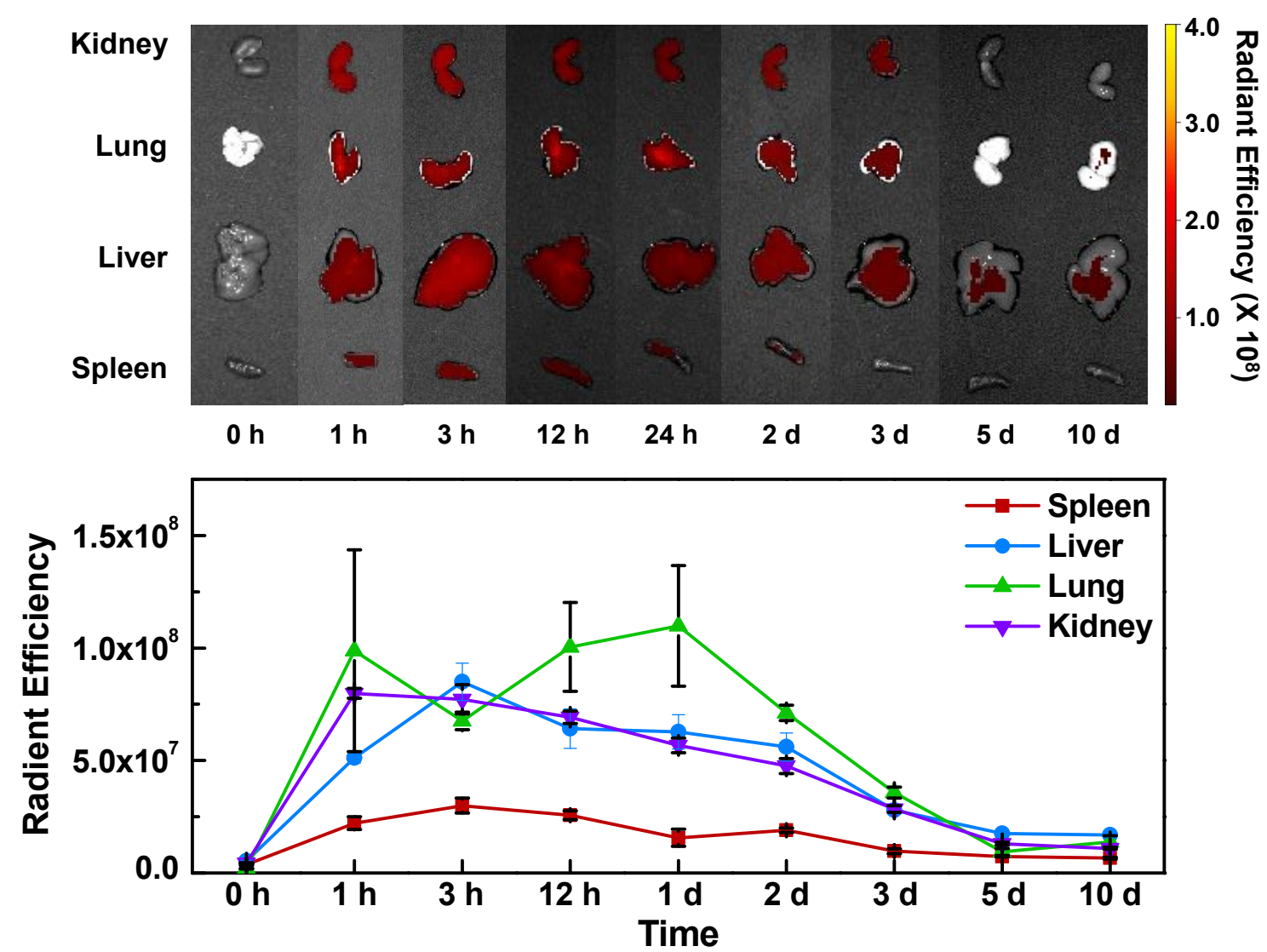

Figure S29. Pharmacokinetics of intraperitoneally injected $\mathrm{WS}_{2}$ nanosheets in vivo. a) Fluorescence images of the kidney, lung, liver, and spleen of the mice intraperitoneally administrated with the $\mathrm{WS}_{2}$ nanosheets bearing IR783 (8.5 $\left.\mathrm{nM}\right)$ and b) Quantitative fluorescence intensities of the organs in (a) measured by an IVIS spectrum-chromatography (CT) system. 


\section{REFERENCES}

(1) Kim, Y. R.; Koh, H. J.; Kim, J. S.; Yun, J. S.; Jang, K.; Lee, J. Y.; Jung, J. U.; Yang, C. S. Peptide Inhibition of p22phox and Rubicon Interaction as a Therapeutic Strategy for Septic Shock. Biomaterials 2016, 101, 47-59.

(2) Yang, C. S.; Kim, J. J.; Kim, T. S.; Lee, P. Y.; Kim, S. Y.; Lee, H. M.; Shin, D. M.; Nguyen, L. T.; Lee, M. S.; Jin, H. S.; Kim, K. K.; Lee, C. H.; Kim, M. H.; Park, S. G.; Kim, J. M.; Choi, H. S.; Jo, E. K. Small Heterodimer Partner Interacts with NLRP3 and Negatively Regulates Activation of the NLRP3 Inflammasome. Nat. Commun. 2015, 6, 6115.

(3) Buchweitz, J. P.; Karmaus, P. W.; Harkema, J. R.; Williams, K. J.; Kaminski, N. E. Modulation of Airway Responses to Influenza A/PR/8/34 by Delta9-Tetrahydrocannabinol in C57BL/6 Mice. J. Pharmacol. Exp. Ther. 2007, 323, 675-683.

(4) Kennedy, R. J.; Hoper, M.; Deodhar, K.; Erwin, P. J.; Kirk, S. J.; Gardiner, K. R. Interleukin 10-Deficient Colitis: New Similarities to Human Inflammatory Bowel Disease. Br. J. Surg. 2000, 87, 1346-1351. 